\title{
Earthquake source characteristics from dynamic rupture with constrained stochastic fault stress
}

\author{
J. Ripperger, ${ }^{1}$ J.-P. Ampuero, ${ }^{1}$ P. M. Mai, ${ }^{1}$ and D. Giardini ${ }^{1}$ \\ Received 18 May 2006; revised 7 November 2006; accepted 29 November 2006; published 27 April 2007.
}

[1] One of the challenging tasks in predicting near-source ground motion for future earthquakes is to anticipate the spatiotemporal evolution of the rupture process. The final size of an event but also its temporal properties (propagation velocity, slip velocity) depend on the distribution of shear stress on the fault plane. Though these incipient stresses are not known for future earthquakes, they might be sufficiently well characterized in a stochastic sense. We examine the evolution of dynamic rupture in numerical models of a fault subjected to heterogeneous stress fields with varying statistical properties. By exploring the parameter space of the stochastic stress characterization for a large number of random realizations we relate generalized properties of the resulting events to the stochastic stress parameters. The nucleation zone of the simulated earthquake ruptures in general has a complex shape, but its average size is found to be independent of the stress field parameterization and is determined only by the material parameters and the friction law. Furthermore, we observe a sharp transition in event size from small to system-wide events, governed mainly by the standard deviation of the stress field. A simplified model based on fracture mechanics is able to explain this transition. Finally, we find that the macroscopic rupture parameters (e.g., moment, moment rate, seismic energy) of our catalog of model quakes are generally consistent with observational data.

Citation: Ripperger, J., J.-P. Ampuero, P. M. Mai, and D. Giardini (2007), Earthquake source characteristics from dynamic rupture with constrained stochastic fault stress, J. Geophys. Res., 112, B04311, doi:10.1029/2006JB004515.

\section{Introduction}

[2] The region close to active earthquake faults is the most challenging to deal with in terms of seismic hazard assessment. Not only is it the zone most likely to experience strong shaking and severe damage, but it is also the region with very large variability in observed ground motion intensities (PGA) [e.g., Shakal et al., 2006]. While part of the ground motion variability can be attributed to local site effects and/or (de-) amplification of waves due to complex geological structure, the contribution of earthquake source complexity to this variability is still not fully understood.

[3] Despite an increasing number of strong motion recordings in recent years, observations for the very nearsource region are still scarce, in particular for large earthquakes. Inferring the details of the source rupture process and its effects on the resulting near-source motions is therefore strongly limited by the available data; this is even more accentuated when it comes to deducing statistical properties of earthquake ruptures. Events like the 1999 Taiwan earthquake generated a wealth of recordings, but this data set still contains information only about one single realization of a large crustal thrust-faulting earthquake that produced extensive surface faulting. On the other hand, recent large strike-slip earthquakes, like the 1999 Izmit and

\footnotetext{
${ }^{1}$ Institute of Geophysics, ETH Zurich, Zurich, Switzerland.
}

Copyright 2007 by the American Geophysical Union. 0148-0227/07/2006JB004515\$09.00 the 2002 Denali earthquakes resulted only in few nearsource recordings. Our ability to study earthquake source complexity and to make statistically sound inferences about its effects on near-source ground motions, using observations alone, is therefore limited.

[4] Nevertheless, inversion of seismic and/or geodetic data of past earthquakes have inevitably shown that earthquakes are complex at all spatiotemporal scales [e.g., Hartzell and Heaton, 1983; Beroza and Spudich, 1988; Wald and Heaton, 1994; Sekiguchi et al., 1996; Delouis et al., 2002]. The potential origins of this imaged source variability are heterogeneities in fault stress, heterogeneities in material and friction parameters and complexity of the fault geometry.

[5] This paper deals with the first possibility, i.e., with heterogeneous stress distributions on the fault plane. Early work on heterogeneous stress focused on generic cases, i.e., simple geometric configurations of high or low stress patches [e.g., Day, 1982; Fukuyama and Madariaga, 2000]. A number of studies have successfully constructed dynamic rupture models with highly heterogeneous stress fields to reproduce data for past events [e.g., Miyatake, 1992a, 1992b; Beroza and Mikumo, 1996; Bouchon, 1997; Olsen et al., 1997; Ide and Takeo, 1997; Nielsen and Olsen, 2000; Peyrat et al., 2001; Zhang et al., 2003]. These lowresolution images of dynamic earthquake rupture allow us to visualize stress heterogeneity on the fault plane, but they still represent only particular realizations of source complexity for a small number of earthquakes. They have not 
been used to attempt a more general, quantitative investigation of stress heterogeneity and its effects on rupture dynamics.

[6] Unfortunately, the parameters and quantities governing the dynamics of an earthquake (e.g., stress on the fault) cannot be determined experimentally prior to an earthquake. What might be estimated with sufficient accuracy, however, are statistical descriptions of the stress field. Oglesby and Day [2002] went in that direction by numerical modeling of dynamic ruptures with various cases of strength variabilities combined with heterogeneous stress, using random stress fields that were constructed in a somewhat ad hoc manner. Their work, however, did not attempt to statistically quantify the effects of different parameterizations of stress variability on the dynamic rupture process and the resulting ground motions. In contrast, earlier work by Andrews [1980, 1981] discusses static and kinematic stochastic models of earthquake rupture, with stress heterogeneity described by a power law decay of its wave number spectrum, and their effects on the radiated wavefield. Following this line of thought and adding some simplifying assumptions (e.g., rupture geometry), Frankel [1991] established some general connections between stress heterogeneity, the characteristics of far-field displacement amplitude spectra and the frequency-size statistics of earthquakes. On the basis of this approach, fractal descriptions of fault slip have been used [e.g., Herrero and Bernard, 1994; Zeng et al., 1994; Gallovic and Brokesova, 2004] for ground motion simulation, while other studies estimate statistical parameters to characterize the heterogeneity of slip [e.g., Mai and Beroza, 2002; Lavallée and Archuleta, 2003; Liu-Zeng et al., 2005; Lavallée et al., 2006] and relate those to the faulting process.

[7] In the present study we employ a concise and quantifiable statistical description of stress distribution in order to examine the dynamic rupture behavior due to different parameterizations of stress heterogeneity. The motivation for this work stems from the preceding discussion and the question of how heterogeneity in the initial stress conditions on the fault plane affects rupture nucleation, propagation and arrest. Under what general conditions does rupture nucleate and propagate and exhibit properties similar to observed earthquakes? Rather than focusing on single events we carry out a large number of fully spontaneous dynamic rupture simulations for many realizations of heterogeneous initial stress fields for a variety of stochastic field parameters. This allows us to investigate ensemble statistics of dynamic rupture under inhomogeneous stresses and to determine general patterns in the response of this system that can then be compared against analytical predictions and observations. It is important to point out that we are not modeling seismicity evolution or full earthquake cycles. Our statistical description represents ensemble statistics of possible fault zone states. These states can either be determined by the maturity of the fault in terms of their geological evolution [e.g., Wesnousky, 1988; Hillers et al., 2007] or can be viewed as a particular state due to the background seismicity just before an impending earthquake.

[8] In contrast to previous works with rather generic characterizations of stress heterogeneity, we adopt a spectral description of initial stress as a self-affine correlated random field with a power law decay at high wave numbers. This approach follows the characterization of slip in past earthquakes by Mai and Beroza [2002] and allows us to generate many different stress patterns that differ in their details (i.e., for each realization) but are statistically identical. In choosing this method, we vary the parameters describing the fractal stress distribution, always retaining some common characteristics of the different classes of heterogeneous input stress fields. Moreover, instead of imposing a nucleation point and size, we apply a simplified approach to mimic tectonic loading, coupled to an algorithm for finding the physically most consistent rupture nucleation region (generally of geometrically complex shape).

[9] We point out that we also fix fracture energy for simplicity since its scale dependency is still actively debated [e.g., Abercrombie and Rice, 2005; Mai et al., 2006], and its nonhomogeneous parameterization, in conjunction with heterogeneous stress, yields a vast parameter space that would be difficult to fully explore. Moreover, we instead attempt to isolate and understand the effects of stress heterogeneity on dynamic rupture before including (potentially correlated) heterogeneous distributions of two (or more) dynamic quantities into the modeling strategy. Within the simplifying assumptions in our dynamic modeling, our technique provides a fully self-consistent physical model for earthquake nucleation and propagation, allowing us to investigate the statistics for a large number of rupture simulations with different parameterizations of stress heterogeneity.

[10] The paper is laid out as follows: In section 2 we introduce the model setup, the numerical scheme, and the stochastic characterization of heterogeneous stress we use. We then present the results for various stress heterogeneity parameterizations in terms of nucleation and propagation behavior of a large set of model quakes; we then investigate the resulting dynamic ruptures in terms of their overall scaling properties, their moment rate functions and radiated seismic energy. This enables us to infer which heterogeneity characterization is perhaps the most plausible when comparing our simulations against observations. Our results suggest that it should be possible to place constraints on stress heterogeneity from independent observations which potentially could help to infer general properties of the rupture process of future earthquakes on a given fault.

\section{Method}

\subsection{Model Setup}

[11] To single out the effects of heterogeneity in shear stress, we restrict our model to relative simplicity. We consider a planar fault embedded in a homogeneous, elastic full space. This setting can be treated numerically very efficiently with a boundary integral method. The dynamic rupture calculations presented here are performed using a spectral boundary integral method coded by Dunham [2005], following the methodology of Geubelle and Rice [1995].

[12] The fault is rectangular and slip on the fault is governed by a linear slip-weakening friction law (Figure 1). The critical slip-weakening distance $D_{c}$ is assumed to be uniform over the whole fault, as are the static yield strength $\tau_{s}$ and frictional sliding strength $\tau_{d}$. By fixing $D_{c}$ in the slipweakening model, along with constant strength drop $\tau_{s}-\tau_{d}$, 
a)

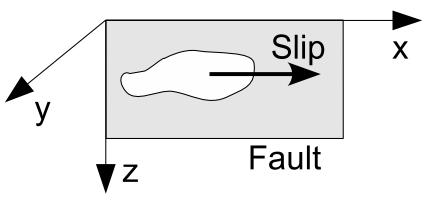

b)

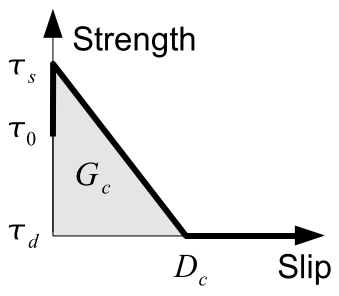

Figure 1. (a) Geometry of the problem. Slip is occurring on a rectangular fault in the xz plane. (b) Linear slipweakening friction law. Once stress has reached the static yield level $\tau_{s}$, strength decreases linearly with slip, until it reaches the dynamic frictional level $\tau_{d}$ at the critical slipweakening distance $D_{c}$. The area under the curve represents fracture energy $G_{c}$.

we constrain fracture energy $G_{c}$ and hence seismic radiation to values roughly consistent for moderate size to large earthquakes $\left(M_{W} \approx 6.4-6.8\right)$, representing a magnitude range particularly important for near-source seismic hazard. In contrast, small earthquakes generated via this approach will be characterized by relatively high fracture energy, resulting in rather low seismic radiation in the higherfrequency range. However, since we focus on studying earthquake dynamics for seismic hazard applications, we deliberately accept the limitations our current modeling strategy places on the smaller magnitude events.

[13] There is no free surface, but the fault is surrounded by unbreakable barriers on all sides, which are modeled by setting $\tau_{s}$ to a practically unreachable value.

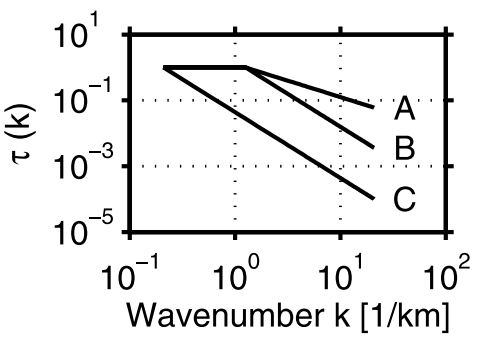

[14] Following Ampuero et al. [2006], we adopt a spectral description of initial shear stress as a self-affine correlated random field with a power law decay at high wave numbers. In the wave number domain the stress field is constrained to a two-dimensional amplitude spectrum $\tau(k)$, where $k=$ $\sqrt{k_{x}^{2}+k_{z}^{2}}$ and $k_{x}, k_{z}$ are the wave number components in the $x$ and $z$ directions, respectively. In particular, the spectrum has a plateau below a given corner wave number $k_{c}$, while above $k_{c}$ the decay is governed by the Hurst exponent $H$ [e.g., Voss, 1988; Mai and Beroza, 2002]:

$$
\tau(k) \propto\left\{\begin{array}{l}
k_{c}^{-(1+H)}=\text { const } \text { for } k \leq k_{c} \\
k^{-(1+H)} \text { for } k>k_{c} .
\end{array}\right.
$$

Particular random realizations are constructed in the Fourier domain by specifying the amplitude according to equation (1) and adding phase values consisting of random numbers uniformly distributed between $[0,2 \pi]$. After performing the two-dimensional inverse Fourier transform under the constraint of Hermitian symmetry, the resulting distributions $\tau(x, z)$ in the space domain (Figure 2) have an approximately Gaussian distribution of amplitude values. The stress distributions are then scaled to a given standard deviation (std) controlling the absolute amplitude variations. Thus, apart from the probability density function (pdf), the random stress fields are controlled by the three parameters $H, a_{c}$ and std, where $a_{c}$ denotes the correlation length $a_{c}=$ $2 \pi / k_{c}$. This parameter also appears in the spectral description of von Karman fields adopted by Mai and Beroza [2002] and

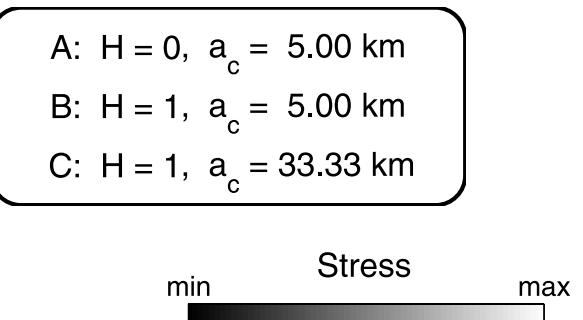

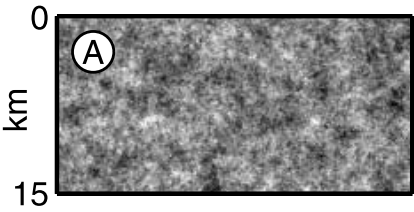
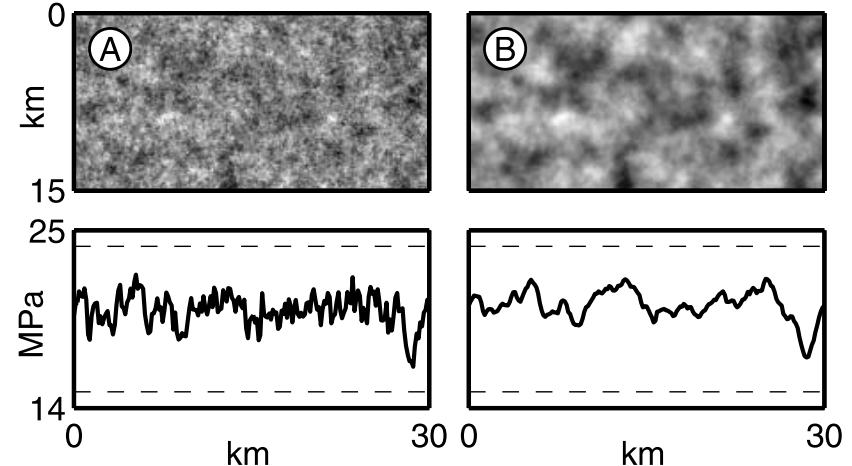
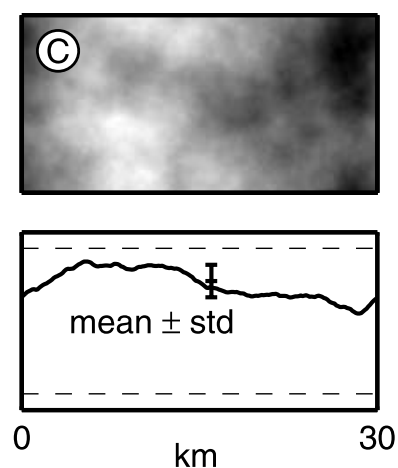

Figure 2. Illustration of random stress field generation. (top) Prescribed Fourier amplitude spectra for three different combinations of Hurst exponent $H$ and correlation length $a_{c}$. Shown are one-dimensional slices (at $k_{z}=0$ ) of the two-dimensional spectra $\tau\left(k_{x}, k_{z}\right)$, normalized to $\max (\tau)=1$. (middle) Example random realizations of initial stress for the wave number spectra given above. (bottom) Along-strike profiles of the stress fields above. Profiles are taken at the center of the fault. Upper and lower dashed lines indicate the yield and frictional stress level, respectively. 
Ampuero et al. [2006]. Variations of these three parameters will be studied in the main part of this paper.

[15] Recent work by Lavallée and Archuleta [2003] and Lavallée et al. [2006] suggests that slip and stress distributions on real faults may follow a non-Gaussian pdf with a broader range of values. A more general class of pdf's allowing for the occurrence of extreme values is provided by the Levy pdf [Lavallée et al., 2006]. However, the Levy distributions are characterized by four parameters instead of the two needed for a Gaussian distribution, thus constituting a higher degree of complexity. Considering the other simplifying assumptions outlined above, we do not consider it necessary to go beyond the simpler case of Gaussian pdf's in this study. In particular the assumption of uniform strength drop limits the admissible range for extreme stress drop values. Accordingly, initial tests with heavy tailed Levy distributions yield either extremely large preslip values (larger than $D_{c}$ ) due to very narrow stress peaks and/or regions where stress is far below the frictional sliding strength. Both cases are rather unlikely to appear on real faults. Additional tests considering uniformly distributed random stress values indicated no significant differences in the model response compared to the case of Gaussian distributions.

\subsection{Loading and Nucleation}

[16] In our modeling approach, we assume that tectonic loading occurs as uniformly increasing shear stress on the fault plane, raising the initial stress field to a critical state, i.e., to a stress state where any further loading leads to dynamic rupture propagation. In this context "dynamic" refers to propagation not driven by tectonic loading anymore, but by the stress changes induced by the propagating rupture itself. We further assume that nucleation can be accurately described as a quasi-static process, also governed by linear slip weakening. This case has been studied analytically for two-dimensional (2-D) in-plane and antiplane ruptures by several researchers [e.g., Campillo and Ionescu, 1997; Uenishi and Rice, 2003]. One remarkable result of the analysis by Uenishi and Rice [2003] is the existence of a critical nucleation length, which depends only on the material properties and the slope of the friction law, but not on the particular shape of the stress function. For the in-plane fracture mode this length is given by

$$
a_{\nu}=1.158 \frac{\mu}{1-\nu} \frac{1}{W} .
$$

where $\mu$ and $v$ are the shear modulus and Poisson ratio of the medium, respectively. For the antiplane fracture mode the term $1-v$ has to be dropped. The slope of the slip-weakening friction law is defined as $W=\left(\tau_{s}-\tau_{d}\right) / D_{c}$, where $\tau_{s}$ and $\tau_{d}$ are the static and dynamic frictional strength, respectively.

[17] Some results for special cases of 3-D ruptures were given by Uenishi and Rice [2004], but there is no analytical solution available for the general 3-D case. Here, we approximate the loading process by finding the critical state of stress (i.e., the last stable stress state just prior to dynamic instability) through an iterative procedure. It involves dynamic rupture calculations for small subsections of the fault plane and is described and discussed in detail in Appendix A. It is important to note that the procedure employed neglects the effects of quasi-static preslip and therefore tends to
Table 1. General Modeling Parameters Used in All Dynamic Rupture Simulations Presented in This Paper ${ }^{\mathrm{a}}$

\begin{tabular}{lcl}
\hline \multicolumn{1}{c}{ Parameter } & Symbol & \multicolumn{1}{c}{ Value } \\
\hline Density & $\rho$ & $2800 \mathrm{~kg} / \mathrm{m}^{3}$ \\
P wave velocity & $v_{p}$ & $6000 \mathrm{~m} / \mathrm{s}$ \\
S wave velocity & $v_{s}$ & $3464 \mathrm{~m} / \mathrm{s}$ \\
Critical slip-weakening distance & $D_{c}$ & $0.2 \mathrm{~m}$ \\
Yield strength & $\tau_{s}$ & $24 \mathrm{MPa}$ \\
Frictional sliding strength & $\tau_{d}$ & $15 \mathrm{MPa}$ \\
Grid spacing & $\Delta x$ & $150 \mathrm{~m}$ \\
Time sampling & $\Delta t$ & $0.013 \mathrm{~s}$ \\
Fault dimensions & & $15 \times 30 \mathrm{~km}$ \\
\hline
\end{tabular}

${ }^{a}$ The only exception being Figure 3 , where some results for $D_{c}=0.4 \mathrm{~m}$ have been added.

underestimate the size of the nucleation zone while overestimating the critical load (i.e., the increase in stress necessary to reach the critical stress state), affecting therefore also the actual values of stress in our numerical results. We have recently developed an improved algorithm to correctly solve the quasi-static problem associated with uniform tectonic loading, including preslip and the resulting stress redistribution (to be presented in a forthcoming paper). Initial results show that our general conclusions are not affected by the bias introduced by the approximating procedure used throughout the present paper.

\section{Parameters and Nondimensional Quantities}

[18] To characterize and generalize the properties of the dynamic rupturing process, we explore the space of parameters quantifying the stochastic shear stress, while the frictional parameters and the fault geometry are kept constant. The general parameters which are not varied in this study are listed in Table 1. We will discuss our results in terms of the following nondimensional quantities:

[19] First, the Hurst exponent $\mathrm{H}$ controls the high wave number falloff of the stress spectrum. It is related to the fractal dimension $D$ by $D=E+1-H$, where $E$ is the Euclidean dimension, and hence for our 2-D distributions $D=3-H$. We consider $H=0,0.5,1$, equivalent to

$$
\left|\tau\left(k>k_{c}\right)\right| \propto k^{-1}, k^{-1.5}, k^{-2} .
$$

This is the same range as discussed by Andrews [1980] for his static stochastic fault model, where $|\tau(k)| \propto k^{-1}$ is required for strict geometrical self-similarity of the ruptures with stress drop being independent of rupture size. By fitting focal mechanism data of earthquakes in southern California, Smith [2006] estimated a 1-D falloff exponent of $\sim 0.8$ for stress, equal to a 2-D falloff $|\tau(k)| \propto k^{-1.3}$ or a Hurst exponent of $H \approx 0.3$, which lies within the range of our parameterization.

[20] The second quantity is given by the ratio $a_{v} / a_{c}$ of the nucleation length $a_{v}$ to the correlation length $a_{c}$ of the stress field. As a proxy for the nucleation length we use the critical length for 2-D in-plane rupture as given by equation (2). For the parameters listed in Table 1 the nucleation length is therefore $a_{v} \approx 1153 \mathrm{~m}$. For the correlation length $a_{c}=2 \pi / k_{c}$ we choose one value on the order of the whole fault length $\left(a_{c}=33.33 \mathrm{~km}\right)$ and one where the correlation length is substantially shorter than the fault dimensions $\left(a_{c}=5 \mathrm{~km}\right)$, thereby yielding $a_{v} / a_{c} \approx 0.035$ and 0.231 , respectively. 
[21] The ratio $\operatorname{std} /\left(\tau_{s}-\tau_{d}\right)$ of the standard deviation of the stress field to the total strength drop constitutes the third nondimensional quantity. From the viewpoint adopted here stress heterogeneity arises from previous seismicity. The amplitude of stress concentrations at the edges of previous ruptures is on the order of $\tau_{s}-\tau_{d}$ and the amplitude of troughs due to dynamic stress overshoot are expected to be less than $30 \%$ of $\tau_{s}-\tau_{d}$. Then, with a fixed value of $\tau_{s}-\tau_{d}$ and an approximately Gaussian distribution of stress values, this would imply a value of $\operatorname{std} /\left(\tau_{s}-\tau_{d}\right)$ smaller than approximately 0.16 . We cover a wider range by varying this ratio from roughly 0.11 to $0.33\left(\tau_{s}-\tau_{d}=9 \mathrm{MPa}, \mathrm{std}=1,2\right.$, $3 \mathrm{MPa}$ ), where the last case may lead to stress troughs unrealistically far below the frictional strength.

[22] In addition, it proves insightful to consider a quantity describing the average stress level at the critical stress state in relation to the total strength drop. We use the nondimensional ratio of average available stress drop to total strength drop

$$
\bar{\tau}_{0}=\left\langle\frac{\tau_{0}-\tau_{d}}{\tau_{s}-\tau_{d}}\right\rangle,
$$

where the angle brackets denote averaging across the fault plane.

\section{Properties of the Dynamic Rupture Process}

[23] Let us now examine the characteristics of nucleation, propagation and arrest for more than 400 dynamic rupture models with variable degrees of stress heterogeneity. We will first investigate the conditions of the critical load and the associated stress levels. The size and shape of the triggering patch, the initiation of rupture, its propagation style and the stress-dependent size transition will be discussed. All these parameters are generally only accessible in numerical simulations, and cannot be easily inferred from observational data.

\subsection{Critical Load}

[24] The critical load $\Delta \tau_{c}^{\infty}$ is defined as the amount of stress which has to be added uniformly to reach the critical stress state (see section 2.2 and Appendix A), starting from a stress state at which the maximum of the shear stress coincides with the yield strength. We expect to see a dependence of the critical load on the ratio $a_{v} / a_{c}$. The smaller the nucleation length $a_{v}$ is in relation to the correlation length $a_{c}$, the less loading is expected to be necessary. For $a_{v} \leq a_{c}$ we empirically find the critical load $\Delta \tau_{c}^{\infty}$ to depend on the ratio $a_{v} / a_{c}$ as

$$
\Delta \tau_{c}^{\infty} \propto \operatorname{std}\left(\frac{a_{\nu}}{a_{c}}\right)^{(H+1) / 2}
$$

Note that the critical load scales linearly with the standard deviation of the stress field std, a result that follows from dimensional analysis when slip is smaller than $D_{c}$, a typical situation during nucleation. These relationships are depicted in Figure 3, where results for a larger value of $D_{c}\left(D_{c}=0.4 \mathrm{~m}\right)$ and thus a larger $a_{v}$ are included to better illustrate the scaling over a broad range. So if the statistical properties of the stress field and the friction law are known or can be approximately inferred from independent observations, these relationships

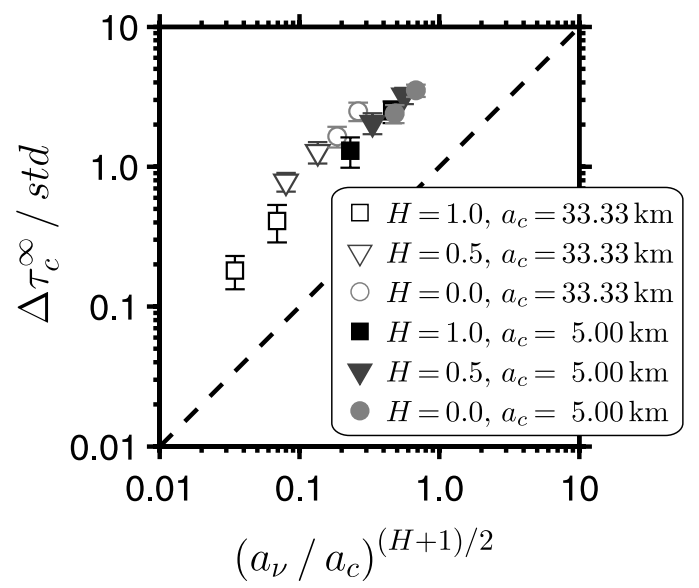

Figure 3. Scaling of critical load $\Delta \tau_{c}^{\infty}$ with ratio $a_{v} / a_{c}$ and $H$. Each symbol and its error bars represent the mean and the standard deviation of 30 random realizations. Since $\Delta \tau_{c}^{\infty}$ is normalized by the standard deviation of the stress field std, results for different std would plot almost exactly on top of each other. Hence only the results for $s t d=1 \mathrm{MPa}$ are shown to improve clarity. Two data points appear for each parameter set because results for $D_{c}=0.2 \mathrm{~m}$ and $D_{c}=$ $0.4 \mathrm{~m}$ are displayed.

provide a good estimate of the amount of tectonic loading necessary to initiate dynamic rupture and of the duration of precursory aseismic slip.

\subsection{Stress Level}

[25] After the tectonic load is applied, stress on the fault is in a critical state, where any additional loading will trigger dynamic rupture. As mentioned in section 3, the average stress level at this critical stress state is expressed by $\bar{\tau}_{0}$, defined as the average ratio between stress drop and total strength drop.

[26] Figure 4 depicts the dependency of this measure on the statistical stress parameters. Two main features can be identified: (1) Stress levels are higher for smaller values of $\operatorname{std} /\left(\tau_{s}-\tau_{d}\right)$, which is the expected behavior, because at the critical stress state, for smaller std all points on the fault will on average be closer to the yield stress than for larger std. (2) Stress levels increase slightly with decreasing Hurst exponent $H$. Stress levels from a reference 2-D analysis, however, do not exhibit the latter dependency, so probably the stress levels are biased toward higher values by our approximative procedure of finding the critical stress state (see section 2.2 and Appendix A).

[27] In either case, the average stress level at the critical stress state is most strongly influenced by the quantity std/ $\left(\tau_{s}-\tau_{d}\right)$, i.e., by the amplitude of the stress heterogeneity. In the following, the stress level will be shown to be a key parameter in our model, controlling the propagation and final size of the ruptures.

\subsection{Triggering Patch}

[28] As mentioned in section 2.2, the ruptures in our simulations are triggered by a patch of the fault with stress slightly above the yield level of the material. The individual triggering patches are found through the iterative procedure described in Appendix A and are ultimately determined by the shape of each particular random realization of the initial 


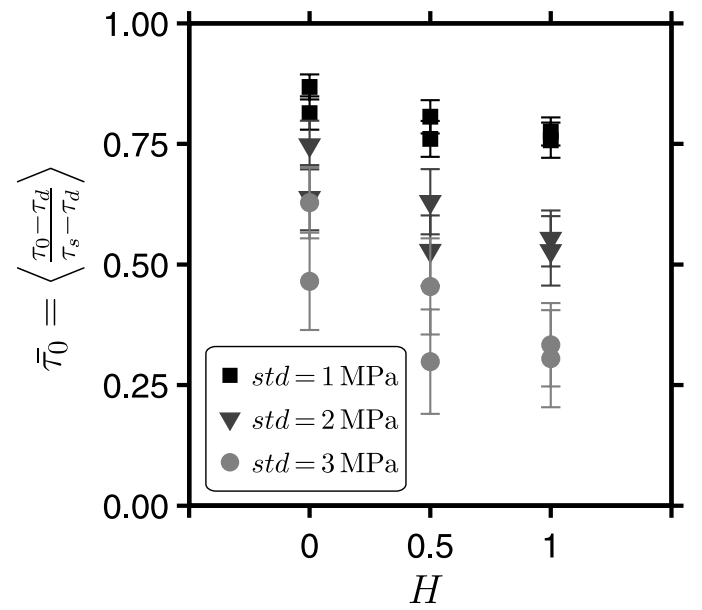

Figure 4. Average stress level $\bar{\tau}_{0}$ versus Hurst exponent $H$ for three different standard deviations. Each symbol and its error bars represent the mean and the standard deviation of 30 random realizations. Note the strong influence of the standard deviation.

stress function. As a first-order characterization of triggering patch size we use the radii of circumscribed and inscribed circles (Figure 5). We find that the inner radius is an approximately constant property over all the parameter variations of the stress field. This confirms that nucleation takes place on a common length scale, depending only on the friction law and the material parameters, similar to what has been shown for the 2-D cases [Uenishi and Rice, 2003]. The critical half lengths for the 2-D cases from equation (2), as well as the critical elliptical radii from equation (A2) are shown in Figure 5 for reference. It can be seen that the radii of the inner fitting circles are coinciding roughly with the critical half length of the in-plane case.

[29] In general, the outer radii show a larger variability within each parameter set, and an increase with decreasing Hurst exponent $H$. The latter reflects the transition from close to circular to more complex patch shapes due to the increase in small-scale complexity in the stress distributions.

\subsection{Initiation of Rupture}

[30] After triggering by the "initial kick" of the stress increment in the triggering patch (see Appendix A), slip velocities remain at rather low levels $(<0.001 \mathrm{~m} / \mathrm{s})$ for some time during which the size of the slipping region does not change much. Eventually an exponential increase in slip velocity marks the transition to fast dynamic propagation. Figure 6 (top) illustrates this exponential seismic nucleation phase (moment rate $\dot{M}_{0}(t) \propto \exp \left(s_{m} t\right)$ ) predicted by Campillo and Ionescu [1997] and Ampuero et al. [2002] and thoroughly discussed by Ampuero and Vilotte [2003]. The characteristic frequency $s_{m}$ of this phase is a robust and observable attribute directly related to a frictional property: It is proportional to the slip weakening rate $W$ :

$$
s_{m}=W /\left(\frac{\mu}{2 v_{S}}\right) \approx 9.3 \mathrm{~Hz}
$$

This attribute can be measured from the initial stages of the waveforms in the same way as the dominant frequency $\omega_{i}$ defined by Allen and Kanamori [2003] or Olson and Allen [2005] for their early warning system strategy (Figure 6, bottom). The dominant frequency $\omega_{i}$ at time step $i$ of our numerical simulation can be defined by

$$
\omega_{i}=\sqrt{\frac{A_{i}}{V_{i}}}
$$

where $V_{i}$ and $A_{i}$ are the squared moment rate and moment acceleration smoothed by a first-order Butterworth filter with cut-off frequency $f^{*}$. In recursive form,

$$
V_{i}=\alpha V_{i-1}+\left(\dot{M}_{0}\right)_{i}^{2}
$$

$$
A_{i}=\alpha A_{i-1}+\left(\ddot{M}_{0}\right)_{i}^{2}
$$

where $\alpha=1-f^{*} \Delta t$ and $\Delta t$ is the time-stepping interval. We take $f^{*}=1 \mathrm{~Hz}$ after Allen and Kanamori [2003]. Shortly after slip has reached $D_{c}$, the dominant frequency
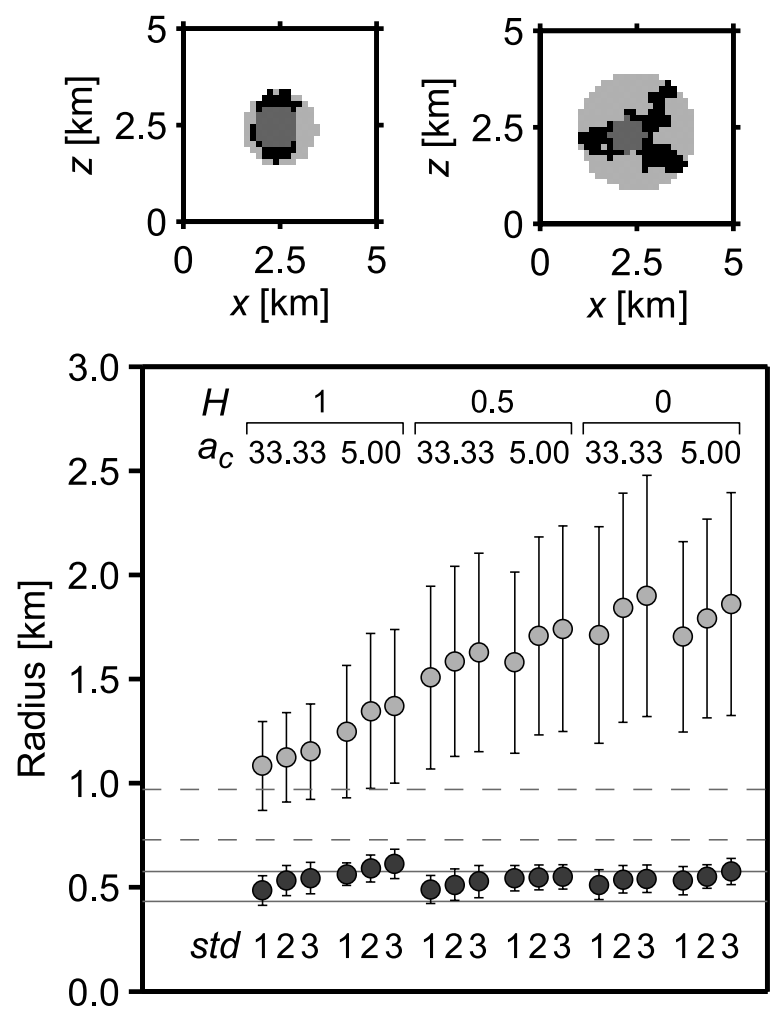

Figure 5. (top) Examples of obtained triggering patches (black) and their circumscribed (light gray) and inscribed (dark gray) circles. (bottom) Radii of circumscribed and inscribed circles. Plotted are the mean radii for 30 random realizations of each parameter set ( $\mathrm{x}$ axis) with error bars of one standard deviation. Solid lines indicate the two 2-D nucleation half lengths for the mode II and mode III cases given in equation (2) and the following discussion, while dashed lines mark the critical elliptic radii of equation (A2). The inner radius is not strongly dependent on the stress field parameterization. 

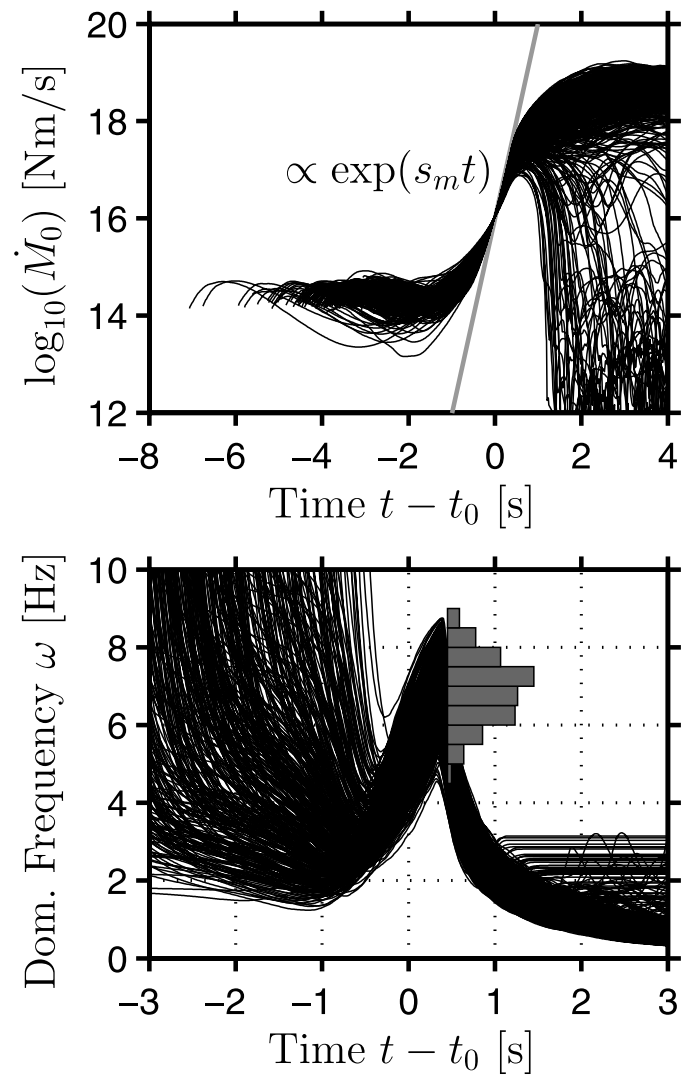

Figure 6. (top) Moment rate functions for all simulations aligned at the time $t_{0}=0$ where moment rate first reaches $10^{16} \mathrm{~N} \mathrm{~m} / \mathrm{s}$. The gray line represents scaling $\propto \exp \left(s_{m} t\right)$ for reference. (bottom) Dominant frequency of the moment rate functions over time. The histogram illustrates its distribution at the (local) maxima.

reaches a maximum. As shown by the histogram in Figure 6 (bottom), measuring $s_{m}$ as the frequency at these local maxima yields a distribution of $s_{m}$ values spanning approximately 4-9 $\mathrm{Hz}$ with a peak around $7 \mathrm{~Hz}$. These values are lower than the value given by equation (6), which would be asymptotically reached for rupture sizes many times larger than the initial nucleation size. In our case, slip reaches $D_{c}$ and nucleation ends before the dominant frequency has reached its asymptotic value, at slipping zone sizes of roughly $2-3$ times the size of the triggering patch. According to Favreau et al. [2002] (in particular their Figure 10), for a quasi-elliptical slipping patch 2-3 times larger than the critical size the dominant frequency is expected to be roughly $0.6-0.7$ times the asymptotic value $s_{m}$, which is in agreement with our numerical observations.

\subsection{Rupture Propagation Style}

[31] One important constraint on the rupture behavior is the choice of the fixed fault size. Since the fault is surrounded by unbreakable barriers, rupture is forced to stop once it hits the fault boundaries. This imposes upper limits on the final size and seismic moment of the events, but also affects rupture propagation: If the rupture nucleates close to one of the boundaries, it will encounter the barrier much earlier on one side than on the other. This effectively leads to unidirectional, occasionally pulse-like, rupture propagation as illustrated in Figure 7. Crack-like propagation prevails for small events not reaching the fault borders and for large events with hypocenters at the center of the fault plane. Conceptually, the propagation style of the pulselike events can be placed somewhere between classical cracks and self-healing slip pulses. This has been previously recognized by Johnson [1990] as a possible mechanism of creating the short slip rise times that have been inferred for real earthquakes [Heaton, 1990]. Interestingly, all seven earthquake source models cited by Heaton [1990] exhibit a distance of the hypocenter from the fault boundary of only about $10-30 \%$ of the total fault dimension, hence favoring unidirectional propagation. In our simulations, the width of the slipping zone (i.e., "pulse width") and the rise time of slip are still large compared to the examples given by Heaton [1990]. However, the largest part of pulse width and risetime is related to very slow slip velocities, so it might be difficult for waveform inversions to distinguish our pulse-like ruptures from self-healing pulses.

[32] The hypocenter position with respect to the fault boundaries is the controlling factor regarding unidirectional or bidirectional propagation. This is in good agreement with results obtained by McGuire et al. [2002], who found that unidirectional propagation dominates for crustal strike-slip events and who were able to explain this observation by a model with hypocenters uniformly distributed over a fault with fixed size, comparable to our simulation setup.

\subsection{Stress-Dependent Size Transition}

[33] For all values of the average stress level $\bar{\tau}_{0}$ higher than a threshold value, the dynamic rupture cannot be stopped by the stress fluctuations and results in a system-wide event. For lower average stress levels we observe a transition to smaller event sizes. This is illustrated in Figure 8, where the average stress level was varied by changing the standard deviation of the initial stress distribution. On average, this transition occurs at average stress levels of 0.3 ,
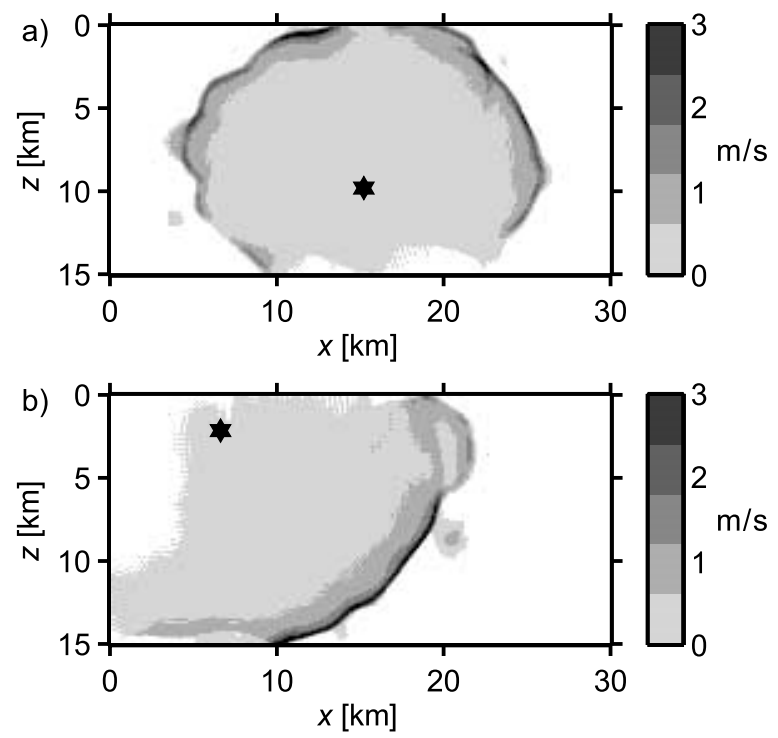

Figure 7. Snapshots of slip velocity on the fault plane for typical examples of (a) crack-like and (b) pulse-like propagation styles. Black stars indicate hypocenters; white areas are not slipping. 

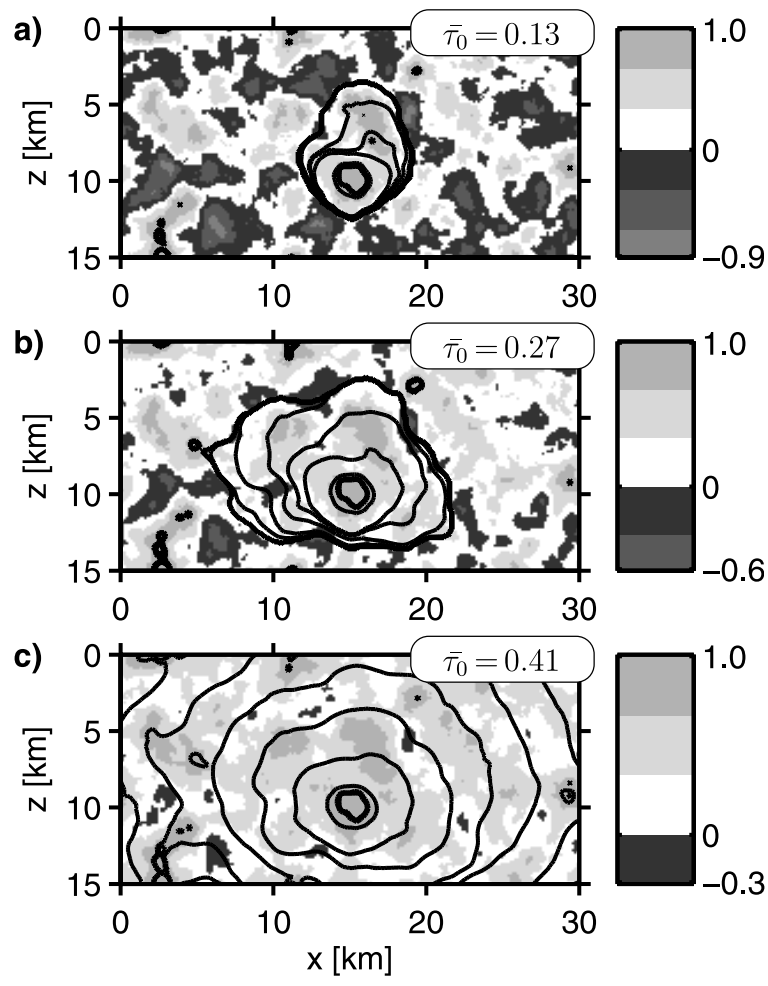

Figure 8. Contours of rupture front (black lines) plotted every second, superimposed on a gray scale map of $\left(\tau_{0}-\right.$ $\left.\tau_{d}\right) /\left(\tau_{s}-\tau_{d}\right)$ across the fault. In these three simulations the average stress level $\bar{\tau}_{0}$ was varied by using different values for the standard deviation of the initial stress $(\mathrm{std}=3,2.5$, and $2 \mathrm{MPa}$ in Figures $8 \mathrm{a}, 8 \mathrm{~b}$, and 8c, respectively). All other parameters are the same in these three examples.

as shown in Figure 9, in which rupture area is plotted as a function of $\bar{\tau}_{0}$ for $H=1$ and $a_{c}=5 \mathrm{~km}$. For other values of $H$ we obtain similar plots, whereas for the cases with $a_{c}=33.33 \mathrm{~km}$ the transition is less clearly visible, because the size of the "smaller" events below the transition is still comparable to the whole fault size. To stop propagating ruptures in our simulations the stress amplitude variations have to be so large that some areas of the fault have initial stress below the frictional strength. On real faults such areas can result from dynamic overshoot of previous events or from aseismic creep. However, this appears to be plausible only, if these areas constitute a minor fraction of the total fault area and overshoot is less than about $30 \%$ of the total strength drop. The threshold value of $\bar{\tau}_{0}=0.3$ translates to roughly $20 \%$ of the fault area being below frictional strength.

[34] Let us explore how final event size is related to the statistical stress parameters averaged over a large ensemble of simulations. We start with an analysis of a mean stress field obtained by averaging over 10,000 random realizations, aligned at their individual maxima. This mean stress fields basically consist of a single peak, the shape of which depends on the autocorrelation function of the individual random realizations. The height of the peak is determined by the standard deviation. The results for this mean field can serve as a reference for the single realizations. The results are overlain in Figure 9 and labeled as "mean field." They highlight the sharpness of the transition to system-wide events.

[35] However, not all events seem to follow the trend outlined by the reference field. A closer look reveals that near the transition additional effects like dynamic triggering and crack interaction become important. These can lead to complex rupture patterns, disconnected slip patches and larger final rupture sizes than predicted by the mean field analysis.

[36] Furthermore, we evaluate the rupture size predictions of a static crack model, based on expressions for circular mode I cracks (see Appendix B). This simplified model is an extension of similar 2-D models discussed by Ampuero et al. [2006], who highlighted the role of the stress heterogeneity amplitude for the transition from moderate to system-wide events. The results of the current simplified 3-D model are included in Figure 9 (labeled "circular"). The model slightly underpredicts the rupture area below the transition but in general the transition itself is well captured.

\section{Implications for Observable Macroscopic Properties}

[37] Previously, we have analyzed the properties of the dynamic rupture process using quantities that are in general only accessible in numerical simulations. However, our approach also allows us to calculate earthquake source parameters that can be inferred from seismic observations. We therefore investigate these macroscopic source parameters, computed for the large number of dynamic rupture models and relate them to observations. For the sourcescaling analysis of small and large events, recall that the model constraints on fracture energy limit the possible range

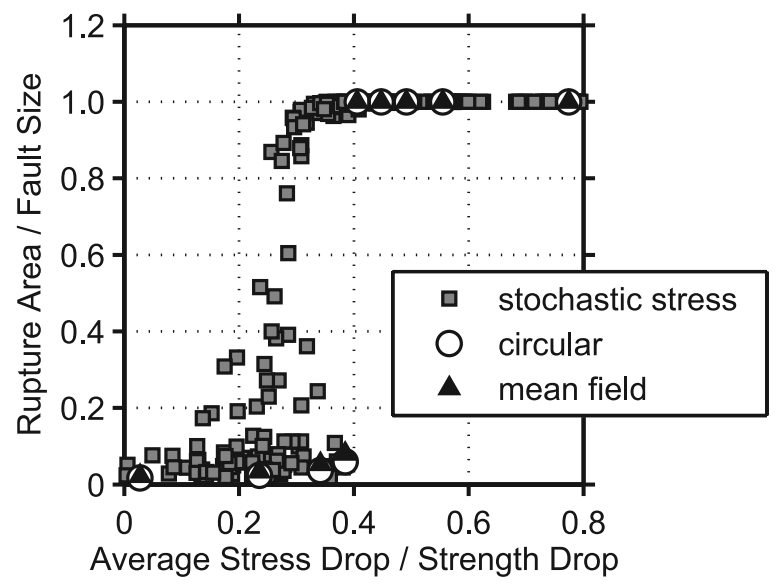

Figure 9. Ruptured area for all simulations with $H=1$ and $a_{c}=5 \mathrm{~km}$ plotted versus average stress level $\bar{\tau}_{0}$, which was varied by changing the standard deviation of the initial stress. Rupture area is normalized by fault size. Triangles represent runs where the initial stress is the mean of 10,000 individual realizations, aligned at their stress maximum. For these averaged stress functions the sharp transition from small to system-wide ruptures is clearly visible. Circles indicate predictions for the mean stress fields of a simplified model assuming circular mode I cracks. 


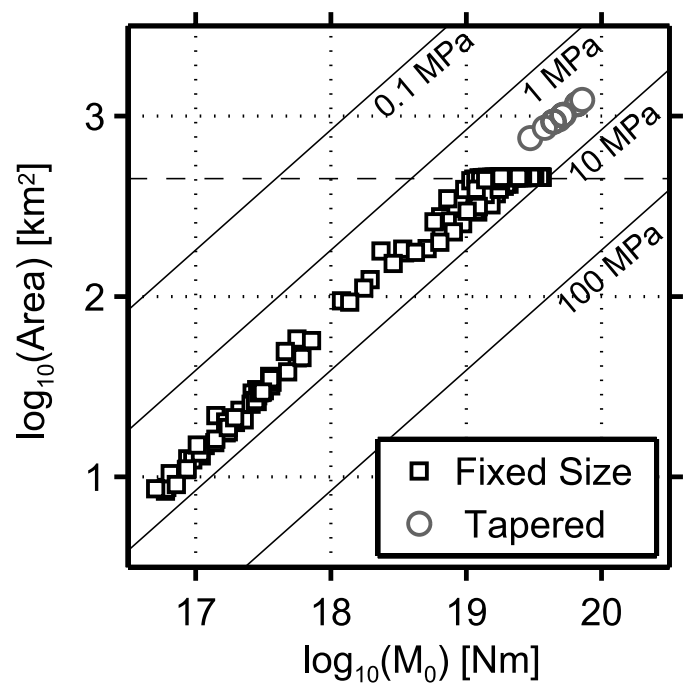

Figure 10. Plot of rupture area versus seismic moment. Note the saturation in the scaling relation at the fixed size of the fault plane at $15 \times 30 \mathrm{~km}=450 \mathrm{~km}^{2}$ (dashed line). A few simulations were performed on a larger fault plane (gray circles). Reference lines are drawn for a scaling of area $\propto M_{0}^{2 / 3}$.

of rupture behaviors and seismic radiation, decreasing in particular the generation and emission of high-frequency energy.

\subsection{Seismic Moment and Radiated Energy}

[38] The events in our simulations span a range of seismic moments of roughly 2.5 orders of magnitude $\left(\sim 7 \times 10^{16}-4 \times\right.$ $10^{19} \mathrm{~N} \mathrm{~m}$ ). Figure 10 displays the scaling of rupture area with seismic moment. Rupture area $S$ here is defined as all the fault area with slip larger than $1 \%$ of the maximum slip. We observe a scaling given approximately by $S \propto M_{0}^{2 / 3}$, comparable to the constant stress drop scaling of observed seismicity [e.g., Kanamori and Anderson, 1975; Wells and Coppersmith, 1994]. At the upper end, the rupture area saturates at the fixed fault size. For a few simulations the assumption of the fixed fault size is relaxed. The fault is enlarged by $9 \mathrm{~km}$ in each direction (i.e., from $15 \times 30$ to $33 \times 48 \mathrm{~km}$ in total) and the unbreakable boundaries are replaced by stress functions slowly decreasing (over $1.5 \mathrm{~km}$ ) to a negative stress drop value of $10 \%$ of the total strength drop. In these cases (gray circles in Figure 10) the $M_{0}^{2 / 3}$ scaling persists.

[39] The total energy $E_{r}$ radiated by the rupture process can be calculated during our simulations in a straightforward manner (see Appendix C). As can be seen in Figure 11, we find that radiated energy scales approximately linearly with seismic moment for all events smaller than the whole fault, thus implying constant apparent stress $\Delta \sigma_{a}$ (the ratio of radiated energy to seismic moment, multiplied by the shear modulus $\left.\Delta \sigma_{a}=\mu E_{r} / M_{0}\right)$. For events spanning the entire fault, radiated energy scales roughly as $E_{r} \propto M_{0}^{3}$, which translates into strongly increasing apparent stress for increasing magnitude. This is generally not observed in real data, and is an effect of the increased radiation due to the abrupt stopping at the unbreakable boundaries (see section 5.2. for further discussion).

\subsection{Moment Rate Spectra}

[40] From our simulations we can readily extract time histories of the total moment release rate. Some examples of moment rate functions for different events are shown in Figure 12. We calculate Fourier amplitude spectra of the moment rate functions which were all zero padded to $2^{13}=$ 8192 samples to enhance frequency resolution. From the spectra we obtain their corner frequency $f_{c}$ and falloff exponent $n$ using the expression [e.g., Abercrombie, 1995]

$$
\Omega(f)=\frac{\Omega_{0}}{1+\left(f / f_{c}\right)^{n}}
$$

and solving for the best fitting set of $\Omega_{0}, f_{c}$ and $n$. For $n=2$ this expression is the spectral shape proposed by Brune [1970]. Fitting is performed in a least squares sense on the logarithm values of the spectrum to reduce biasing by the large values at low frequencies. The fitting range was restricted to frequencies between zero and

$$
f_{\max }=\frac{v_{s}}{4 \Delta x}=\frac{3464 \mathrm{~m} / \mathrm{s}}{4 \times 150 \mathrm{~m}} \approx 5.77 \mathrm{~Hz}
$$

where $\Delta x$ is the spatial grid sampling and $v_{s}$ is the shear wave velocity of the medium. Numerical noise is clearly observed in most cases for frequencies larger than $9 \mathrm{~Hz}$, so we are confident that the fitting procedure is not biased by these high-frequency oscillations. Figure 13 shows amplitude spectra of the example moment rate functions from Figure 12 plus the corresponding fitted spectra.

[41] In all cases the plateau value $\Omega_{0}$ of the spectrum obtained in the fitting process exactly reproduces the seismic moment $M_{0}$ computed from the final slip distribution. The estimated corner frequencies for all events are plotted as a function of seismic moment in Figure 14a. They scale with seismic moment approximately as $f_{c} \propto M_{0}^{1 / 3}$ as expected for crack models of rupture. We cannot find a

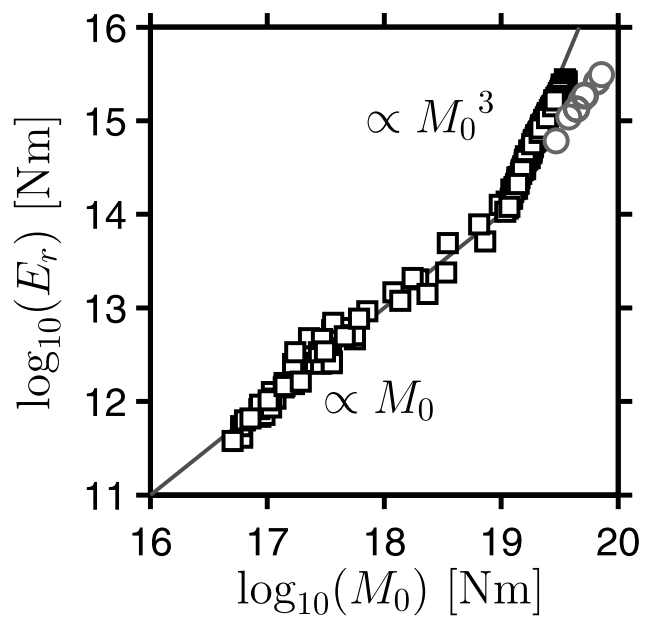

Figure 11. Scaling of radiated energy with seismic moment for all simulations with $a_{c}=5 \mathrm{~km}$. Reference lines are drawn in gray to highlight the change from $E_{r} \propto$ $M_{0}$ to $E_{r} \propto M_{0}{ }^{3}$ when the fixed fault size is reached at around $M_{0}=10^{19} \mathrm{~N} \mathrm{~m}$. This change in scaling is partially due to enhanced radiation, when rupture is stopped abruptly at the fixed fault boundaries. 


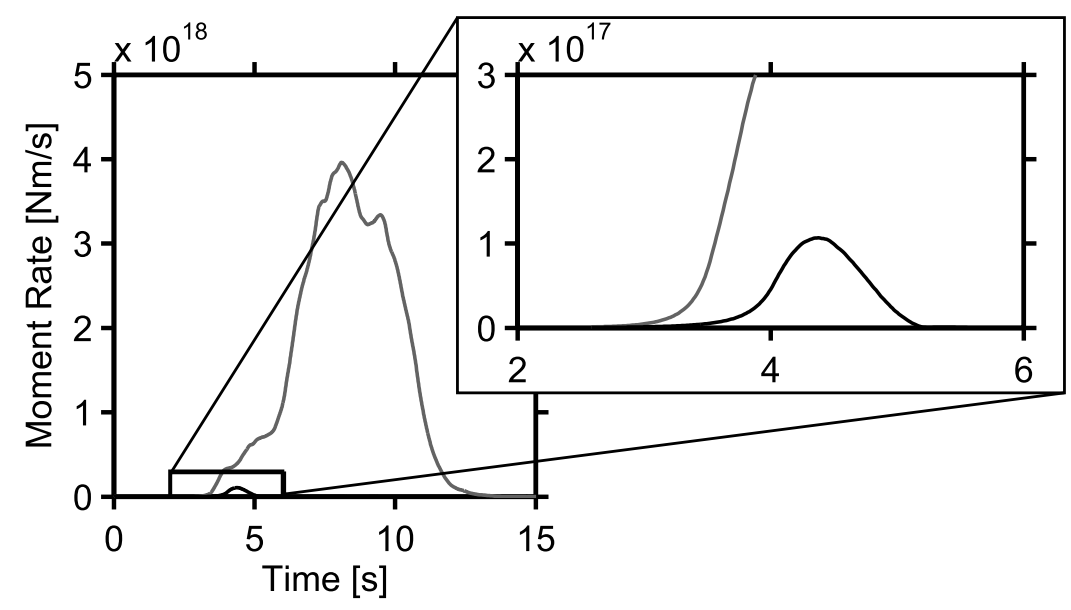

Figure 12. Typical examples of moment rate functions for one of the smallest and one of the largest events.

systematic deviation from this scaling for the pulse-like ruptures, in accordance with the interpretation that these events are not self-healing pulses. An alternative explanation could be, as mentioned above, that the width of the "pulse" is too large to yield a corner frequency significantly different from that of a crack-like rupture.

[42] Figure 14b displays the results for the spectral falloff exponents. For all the events spanning the whole fault size, the exponent $n$ is roughly $2-2.5$. For smaller events, however, we find significantly higher exponents, clustering around $n=3$, corresponding to a steeper high-frequency falloff.

[43] As pointed out by Madariaga [1977], high-frequency radiation is dominated by strong changes in rupture velocity and a lower bound for the spectral falloff exponent is given by $n=2$ for sudden jumps in rupture velocity, e.g., instantaneous stopping of a crack occurring simultaneously along its front. Madariaga [1977] also notes that for abrupt, but not simultaneous stopping, the exponent $n$ will become higher. In addition, Dahlen [1974] considered a crack model including self-similar nucleation and smooth stopping for which he derived a high-frequency asymptotic limit with $n=3$.

[44] These findings offer a consistent explanation for the different spectral falloffs of our simulated ruptures. Nucleation in our model is similar for small and large events and it is a slow and smooth process, not expected to generate significant high-frequency radiation. The small events are strongly affected by our simplistic assumption of constant fracture energy. Their fracture energy is relatively high compared to the energy available to drive the rupture and the events are therefore characterized by unusually low average rupture velocities of roughly $20 \%$ of the shear wave velocity. In addition, they stop early and smoothly by propagating into regions of the fault with low initial stress. Thus, overall, the small events experience no strong changes in rupture velocity and little high-frequency radiation is excited. In contrast, the large events accelerate to higher average rupture velocities before they are abruptly stopped by hitting the unbreakable boundaries, exciting strong stopping phases that increase the high-frequency radiation.

[45] We have repeated a number of simulations in which the unbreakable boundaries are replaced by a slowly decay- ing stress field. These tapered stress fields also stop the ruptures, but over a broader zone and thus more gently. Compared to their untapered counterparts, these events show increased spectral exponents of 2.5-3 (gray circles in Figure 14b). This confirms that a large part of the highfrequency radiation in our dynamic simulations originates from abrupt stopping of the ruptures at the fault boundaries.

[46] In addition, the larger ruptures might generate radiation by repeated acceleration and deceleration due to the heterogeneity in initial stress. However, we do not observe systematic changes of the exponent $n$ with the parameters describing the stress distribution. Probably because most of the ruptures hit the unbreakable boundaries and the resulting stopping phases mask differences in radiation during propagation.

[47] Also, the abrupt fluctuations of rupture velocity required for sustained high-frequency radiation $(n=2)$ are

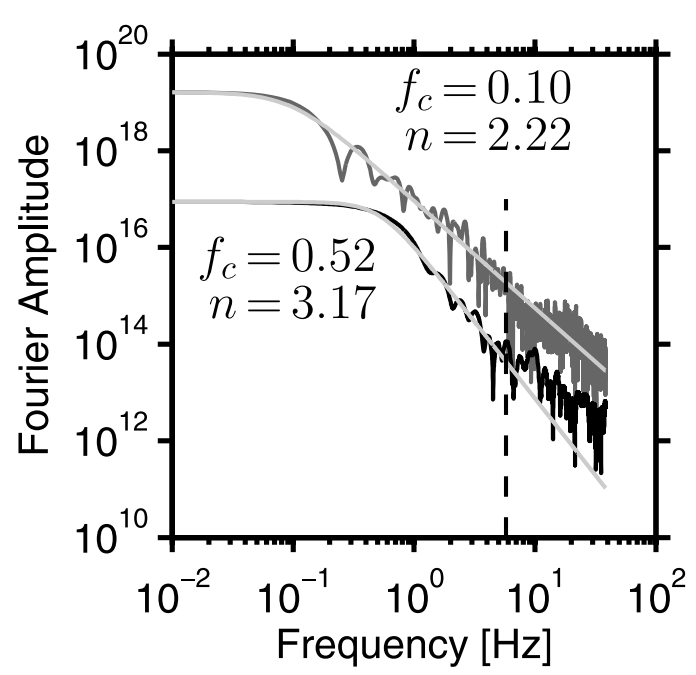

Figure 13. Spectra of moment rate functions for the examples in Figure 12. Their best fitting model spectra are overlain in light gray, along with the corresponding values of corner frequency and spectral falloff. The vertical dashed line marks the upper limit of the spectral fitting procedure of roughly $5.8 \mathrm{~Hz}$. 

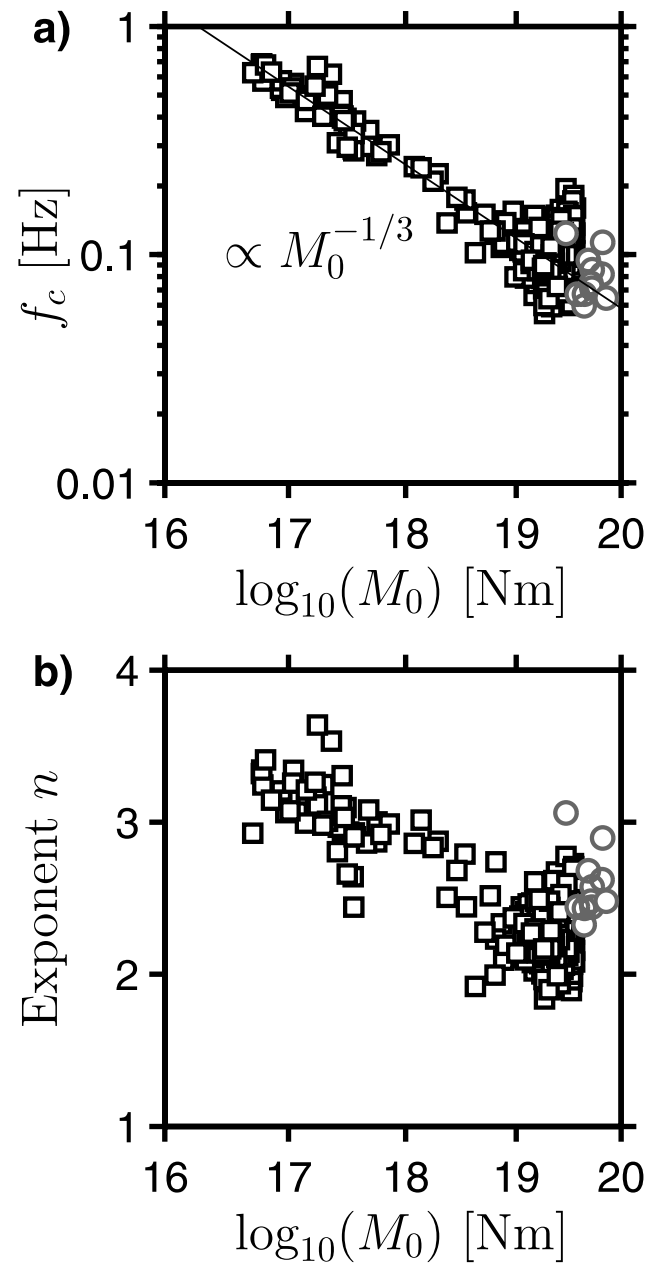

Figure 14. Spectral parameters of moment rate functions for all simulations plotted against seismic moment. (a) Corner frequencies. They show the expected scaling with seismic moment of $f_{c} \propto M_{0}{ }^{-1 / 3}$ indicated by the black reference scaling line. (b) Spectral falloff. Note that the small events and the smoother stopping large events with stress tapered at the edges (gray circles) show falloff exponents $n$ clearly higher than 2 .

less efficiently generated by stress heterogeneities than by heterogeneities of fracture energy, which are not included here. The reason is that, whereas $G_{c}$ enters the "crack tip equation of motion" by its local value, stress drop contributes as a weighted spatial average through the integral defining the stress intensity factor. More singular stress distributions than the ones considered here, such as stress concentrations at the edges of previous events, might be required to generate high-frequency radiation [Madariaga, 1983].

\subsection{Hypocenter Location and Slip Distribution}

[48] In general, the distributions of final slip in our simulations reflect the heterogeneity of the initial shear stress. In particular, stress fields with high Hurst exponent, long correlation length and small standard deviation yield "smoother" distributions, while "roughness" of slip increases with lower Hurst exponent, shorter correlation length and larger standard deviation of stress.
[49] All events not reaching the fault boundaries consist of a single slip patch with the hypocenter located within the region of highest slip (i.e., within the zone of slip $d$ larger than $2 / 3$ of the maximum slip $d_{\max }$ ), often directly coinciding with the location of $d_{\max }$. For the larger events the artificially imposed fault boundaries become important. If ruptures nucleate in the central part of the fault (more than $5 \mathrm{~km}$ distance from any boundary), the hypocenters are still close to the region of highest slip and in more than $95 \%$ of the events hypocentral slip is larger than $2 / 3 d_{\max }$. In contrast, hypocenters located very close to the boundaries (distance to boundary $<2 \mathrm{~km}$ ) are in general more remote from the location of largest slip and only $\sim 7 \%$ of these events have hypocentral slip $d>2 / 3 d_{\max }$. However, for most of these events hypocentral slip is still large $(88 \%$ have $\left.1 / 3 d_{\max }<d<2 / 3 d_{\max }\right)$. So overall the stress load determined hypocenter positions in our simulations are compatible with the hypocenter locations in imaged finite source rupture models [Mai et al., 2005], where it is found that hypocenters are located within or close to regions of large slip.

\section{Discussion}

[50] Let us investigate how our numerical simulations compare against analytical predictions and observations of real earthquakes.

\subsection{Nucleation}

[51] In this study we have assumed that linear slipweakening friction is also the governing process during quasi-static nucleation. Our results on nucleation agree well with and extend earlier studies based on the same assumption [e.g., Campillo and Ionescu, 1997; Favreau et al., 2002; Uenishi and Rice, 2003; Ampuero and Vilotte, 2003]. In particular the common length scale of the nucleation zone (section 4.3) and the exponential growth during the nucleation phase (section 4.4) match well the expectation.

[52] Olson and Allen [2005] observed a magnitude dependency of the dominant frequency measured from the first three seconds of seismograms, which cannot be explained by our assumed constant $W$. It may require a power law nonlinear slip-weakening model [Cochard et al., 2006] or a fault with heterogeneous $D_{c}$ where larger events show some tendency to nucleate in regions of longer critical slip distance (as defined in rate-and-state friction models [Hillers et al., 2006, Figure 14]).

\subsection{Rupture Arrest}

[53] We find that the average final sizes of the ruptures are predicted reasonably well by a simplified model based on fracture mechanical considerations of circular mode I cracks (section 4.6 and Appendix B), constituting a threedimensional extension of the 2-D model by Ampuero et al. [2006]. This simplified model may serve as a guide in choosing stress parameterizations for future scenario calculations, e.g., for a given target event size.

\subsection{Fracture Energy}

[54] In our present approach, both strength drop and $D_{c}$ are constant and hence fracture energy (i.e., the energy dissipated at the tip of the rupture) is also uniform across the 
fault plane and independent of rupture size. However, several studies have indicated a scale dependence of fracture energy [e.g., Ohnaka, 2003; McGarr et al., 2004; Mai et al., 2006]. Though the origin and the details of this scale dependence are still a subject of ongoing debate, it is clear that the assumption of homogeneous fracture energy is a strong simplification. We consider it justified from the viewpoint that an integral aspect of our work is to explore and demonstrate ways of dealing with stochastic descriptions of stress in a generic sense and to estimate the firstorder response of a complex system. The simulations presented in this paper should thus be understood as a starting point for more refined and complex modeling to be guided by and to be built on the current results.

[55] Several ways of including scale-dependent fracture into numerical simulations have been suggested: Aochi and Ide [2004] proposed a renormalization approach, while Andrews [2005] demonstrated that the scale-dependent nonelastic behavior off the fault plane can be mimicked by simply limiting the slip velocity in an elastic medium. Other ways to include scale-dependent fracture energy in our simulations could be a parameterization of $D_{c}$ depending on hypocentral distance and/or stress heterogeneity or the use of a slip-weakening friction law in which strength continues to drop as a small power of slip as suggested by Abercrombie and Rice [2005].

[56] We expect the following changes to our results for scale-dependent fracture energy. The rupture velocity and the seismic radiation properties of the smaller events will better match observational data. Moreover, we expect it to become easier for ruptures to stop with smaller amplitude variations in initial stress and fewer areas (or none) of initial stress below $\tau_{d}$.

[57] Finally, we would like to point out that including systematic scale dependencies of $G_{c}$ into the analysis of Appendix B is straightforward. The scale dependencies discussed in recent literature [e.g., Abercrombie and Rice, 2005; Andrews, 2005] would yield a transition in event size similar to the one described above, but at a different average stress level (see Appendix B).

\subsection{Slip Pulses}

[58] Heaton [1990] pointed out that in many source inversions of real earthquakes slip rise times have to be significantly shorter than the overall event duration to obtain an acceptable fit to the recorded seismograms. As an explanation, Heaton [1990] proposed a model in which velocity-weakening friction results in self-healing pulses of slip propagating over the rupture plane. As discussed in section 4.5, despite crack-like rupture propagation modes are prevailing in our simulations, some of the events show pulse-like features resulting from nucleation close to the fault boundaries. Assuming that because of their limited resolution, waveform inversions might not be able to distinguish between these pulse-like ruptures and selfhealing pulses, this mechanism could offer an alternative explanation for the short rise times of slip inferred for real earthquakes. Alternatively, extending our current model with some healing mechanism is expected to enhance the generation of rupture pulses, probably with even shorter rise times.

\subsection{High-Frequency Radiation}

[59] Our results on the spectral falloff underline the importance of the stopping of earthquake ruptures. Do they just slow down gently, or come to a halt abruptly? Our simulations document how these different mechanisms lead to variations in the high-frequency falloff. The spectral falloff with $n=3$ of the smallest simulated events is generally not observed for real earthquakes in this magnitude range, but is probably a result of the assumed scale independence of fracture energy. However, this case (fracture energy being large compared to the strain energy driving the rupture) may in some cases occur at a much lower magnitude level. Studies on microearthquakes recorded at borehole stations do indeed show a certain variability in the spectral falloff exponents. Abercrombie [1995] states that $n=2$ represents a good average, but also notes that the individual falloff exponents show a significant scatter up to around $n=3.5$. A similar range of values for $n$ was reported by Venkataraman et al. [2006]. In one area they even found a systematic increase in falloff exponents with decreasing event size.

\subsection{Fault Boundary}

[60] In general, all our simulated events yield macroscopic rupture properties in a physically reasonable range. However, the scaling relations of these properties are only compatible with observational data for events smaller than the whole fault size. For the whole fault events the unbreakable fault boundary has been shown to strongly affect the scaling of area, moment and radiated energy. Hence replacing this strong discontinuity in strength by a more gradual change will improve the match of observed scaling relations.

\subsection{Plausible Stress Characterization}

[61] For stress distributions with the lowest standard deviation $\left(\operatorname{std} /\left(\tau_{s}-\tau_{d}\right) \approx 0.1\right)$ we always obtain whole fault events with supershear rupture propagation over much of the fault plane. Such an abundance of supershear propagation is not observed in real earthquakes, rendering these stress models implausible in conjunction with the assumption of homogeneous fracture energy. If fracture energy is heterogeneous, however, a stress distribution with smallamplitude variations or even homogeneous stress also can produce globally subshear ruptures, as demonstrated by Ide and Aochi [2005]. On the other hand, extremely high values of the standard deviation $\left(\mathrm{std} /\left(\tau_{s}-\tau_{d}\right)>0.4\right)$ always lead to the rupture of a single high-stress patch, not being able to propagate to a neighboring patch. These single asperity ruptures generate a single isolated slip patch, not comparable to many of the imaged slip distributions of large earthquakes. Our results therefore suggest that (at least in the simplistic case of homogeneous fracture energy) a standard deviation of stress in the intermediate range is most plausible. As shown in section 4.6, the most complex rupture behavior (including dynamic triggering and rupture jumping) is observed close to the stress-dependent transition in event size.

[62] The stress distributions with correlation length on the order of the total fault dimension also yield slip distributions with a single high-slip patch only. Therefore correlation lengths substantially smaller than the fault size are needed 
to generate the complexity in imaged slip distributions with multiple high-slip patches [e.g., Mai and Beroza, 2002].

\section{Conclusions}

[63] We have investigated the effects of correlated random initial stress on dynamic earthquake rupture. This work establishes links between statistical properties of these stress fields, the governing friction law on the fault and macroscopic rupture properties. Although the details of the actual stress field on a real fault are not accessible to direct measurement, its statistical properties might be reasonably well estimated. Our results provide insights into how these statistical characterizations of fault stress affect the overall rupture behavior and seismic observables. In this context, not only the average macroscopic rupture properties, but also their variability, will be important input to scenario calculations for improved seismic hazard estimates in the near-fault region.

\section{Appendix A: Approximation of Tectonic Loading}

[64] As noted in section 2.2, we approximate tectonic loading and quasi-static nucleation by an iterative procedure. We start with a heterogeneous stress distribution $\tau(x, z)$, where the maximum of the stress distribution has just reached the static yield level $\tau_{s}$ of the material. The main task is to find the critical load $\Delta \tau_{c}^{\infty}$, which is the amount of tectonic stress increase $\Delta \tau^{\infty}$ necessary to bring the fault to the critical stress state at the onset of dynamic instability. Our method of finding $\Delta \tau_{c}^{\infty}$ consists of the following steps:

[65] 1. A starting value for $\Delta \tau^{\infty}$ is chosen.

[66] 2. The set of grid points is determined for which the original stress $\tau(x, z)$ plus the tectonic load exceeds the static yield stress level of the material; that is, we find all grid coordinates $x, z$ for which $\tau(x, z)+\Delta \tau^{\infty} \geq \tau_{s}$.

[67] 3. Within the set of grid points found in step 2 we search for connected clusters of points. Two grid points are defined as connected if they are direct neighbors either in the $\mathrm{x}$ or $\mathrm{z}$ directions. The largest cluster is defined as the one containing the most grid points and will be termed the "triggering patch." At all points belonging to the triggering patch, shear stress will be set to a small value $\tau_{\text {trig }}$ above the yield stress to initiate rupture in the dynamic simulation. The value of $\tau_{\text {trig }}$ should be sufficiently small to still be in accord with the notion of nucleation originating from quasi-statically growing slipping zones. On the other hand it does not have to be smaller than daily variations of stress superimposed on the tectonic load, where Coulomb stresses from Earth tides can be of the order of a few $\mathrm{kPa}$ [Cochran et al., 2004]. Still, the choice of $\tau_{\text {trig }}$ is somewhat arbitrary and we have experimented with different values between 0.1 and $10 \mathrm{kPa}$. Since results only varied slightly, we have chosen $\tau_{\text {trig }}=10 \mathrm{kPa}$ for all the results presented in this paper.

[68] 4. To avoid simultaneous triggering at multiple locations, stress is set to a small value $\tau_{\text {reduce }}$ below yield stress at all remaining points found in step 2 which do not belong to the triggering patch. Since preslip at those points is expected to be small, stress should not have dropped very much at those points and we use a value of $\tau_{\text {reduce }}=10 \mathrm{kPa}$ for all our simulations. However, we do not expect a strong influence for variations of this value.

[69] 5. We carry out a dynamic simulation with the resulting shear stress. To save computation time, the simulation is restricted to a small subsection $(9 \mathrm{~km} \times 9 \mathrm{~km})$ of the fault surrounding the triggering patch and to $\sim 5 \mathrm{~s}$ simulated time. After this duration, in all our simulations either slip has stopped completely on the whole fault or a slip velocity threshold of $0.001 \mathrm{~m} / \mathrm{s}$ has been exceeded, indicating continued rupture expansion.

[70] 6. If slip velocity during this simulation has not reached the threshold value of $0.001 \mathrm{~m} / \mathrm{s}$, we start over at step 1 with an increased value of $\Delta \tau^{\infty}$. If the slip velocity threshold has been exceeded, we start over at step 1 with a decreased value of $\Delta \tau^{\infty}$

[71] Employing this procedure iteratively, we finally obtain the smallest load that still leads to sustained rupture, i.e., an approximated value of the critical load $\Delta \tau_{c}^{\infty}$. Using this critical load, we go through steps $2-4$ of the above procedure to obtain the critical state of the stress field. This stress distribution is used as the initial stress $\tau_{0}(x, z)$ in the dynamic rupture computation on the entire fault. Rupture in this whole-fault dynamic simulation is triggered by the small increment $\tau_{\text {trig }}$ above yield stress inside the triggering patch.

[72] We test the scheme described above, first for one of the 3-D cases treated analytically by Uenishi and Rice [2004]. The stress function is pure shear in the in-plane direction $(x)$ and is given by

$$
\tau(x, z)=\tau_{s}-\frac{1}{2}\left[x^{2}+\left(\frac{1}{1-\nu}\right)^{2} z^{2}\right] .
$$

According to Uenishi and Rice [2004], for homogeneous loading of this stress function, the slipping patch always has an elliptical shape with constant aspect ratio. For $v=0.25$ they provide the following approximate values of the critical elliptical radii $a_{\mathrm{e}}$ and $b_{\mathrm{e}}$ :

$$
\begin{aligned}
& 2 a_{\mathrm{e}} \approx 2.598 \frac{\mu}{W}, \\
& 2 b_{\mathrm{e}} \approx 1.951 \frac{\mu}{W},
\end{aligned}
$$

Applying our loading procedure (with grid spacing $\Delta x=$ $150 \mathrm{~m}$ and triggering stress $\tau_{\text {trig }}=10 \mathrm{kPa}$ ) to this stress function, we compare the obtained results with the theoretical prediction, as shown in Figure A1. The size of the triggering patch is smaller than predicted (elliptical radii of approximately $0.8 a_{\mathrm{e}}$ and $0.8 b_{\mathrm{e}}$ ), whereas the critical load $\Delta \tau_{c}^{\infty}$ is overestimated by a factor of about 2 . This is due to the fact that our approximation neglects the effect of quasistatic preslip. Stress starts to decrease at the location of this quasi-static preslip, but at the same time stress is increased in the surrounding regions, effectively leading to earlier nucleation (i.e., with less loading necessary). If successively smaller increments $\tau_{\text {trig }}$ inside the triggering patch are used, its size tends asymptotically toward the theoretical prediction, but the overestimation of the critical load is increased. So in choosing the triggering stress $\tau_{\text {trig }}$ there is a trade-off between the errors of the approximation of nucleation size and critical load. 


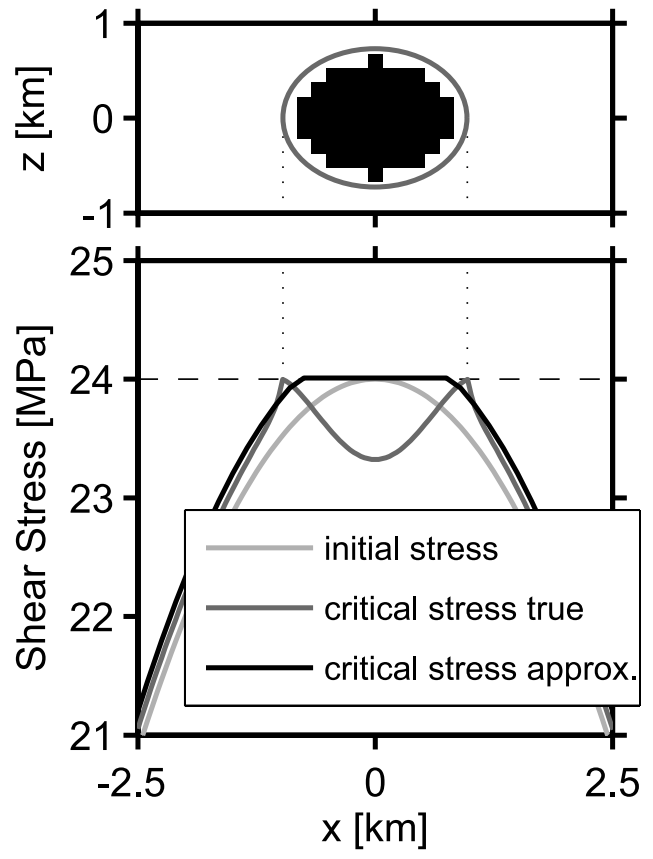

Figure A1. (top) Triggering patch (black) obtained through the approximative loading procedure for the stress function of equation (A1) compared to the theoretical prediction (dark gray ellipse) for a grid spacing of $150 \mathrm{~m}$. (bottom) Stress profiles along the in-plane direction. Shown are the initial stress (light gray), the correct critical stress state including the drop of stress in the center due to quasistatic preslip (dark gray) and the approximation of the critical stress state (black). Dotted lines indicate theoretical 3-D nucleation length. Horizontal dashed line indicates yield strength.

[73] As mentioned in section 2.2 we have developed an improved algorithm (to be presented in a forthcoming paper) to compute the critical load. This enabled us to perform a similar comparison also for the heterogeneous stress distributions, where no analytical solution is available. We find that the critical load is overestimated by our approximation procedure by factors ranging from 1.4 to 1.8 with an average of 1.6. This affects the ratio between stress standard deviation std and average stress level $\bar{\tau}_{0}$ and therefore the values in Figure 4 are slightly shifted upward with respect to their true value. However, the scaling relation of the critical load given by equation (5) remains valid, as do the general results obtained in this paper.

\section{Appendix B: Analysis of the Transition to Runaway Ruptures}

[74] We propose here a mechanical interpretation of the transition from small to fault-wide earthquake sizes illustrated in Figures 8 and 9. This explanation is based on fracture mechanics concepts and is largely inspired by the work of Dyskin [1999]. It extends the discussion presented by Ampuero et al. [2006]. A number of approximations are made that are ultimately tested by comparison to the average properties of our dynamic simulations (Figure 9).
[75] The evolution of a slip-weakening crack with a relatively small process zone can be fairly well described by the Griffith criterion and small-scale-yielding fracture mechanics. Upon arrest, the dynamic stress intensity factor $K$ shows damped oscillations around its static value, generated by multiple wave diffractions along the rupture front. In a rough approximation, the final earthquake size is given by the following arrest criterion (e.g., in the mode II direction):

$$
\frac{1-\nu}{2 \mu} K_{0}^{2}=G_{c}
$$

where $K_{0}$ is the static stress intensity factor and fracture energy is given by $G_{c}=\left(\tau_{s}-\tau_{d}\right) D_{c} / 2$. Realistic rupture fronts have complicated geometry, but for the sake of mathematical tractability we employ the expression of $K_{0}$ for circular mode I cracks. This approximation is justified by our interest in statistically averaged properties arising from isotropic stress drop distributions, and by considering the anisotropy of the shear modes as a second-order effect. For a crack of radius $a$ and a heterogeneous stress drop $\Delta \tau$, it is given in cylindrical coordinates $(r, \theta)$ by Lai et al. [2002], after Fabrikant [1989], as

$$
K_{0}(a, \theta)=\frac{1}{\pi \sqrt{\pi a}} \int_{0}^{2 \pi} \int_{0}^{a} \frac{\Delta \tau(r, \phi) \sqrt{a^{2}-r^{2}}}{a^{2}+r^{2}-2 a r \cos (\theta-\phi)} r d r d \phi .
$$

Clearly, $K_{0}$ fluctuates along the crack rim, but we will assume that the arrest condition can be applied to the average stress intensity factor

$$
K_{0}(a)=\frac{1}{2 \pi} \int_{0}^{2 \pi} K(\theta) d \theta
$$

which can be simplified to

$$
K_{0}(a)=\frac{1}{\pi \sqrt{\pi a}} \int_{0}^{2 \pi} \int_{0}^{a} \frac{\Delta \tau(r, \phi)}{\sqrt{a^{2}-r^{2}}} r d r d \phi .
$$

To estimate the average event-size we first compute $K_{0}(a)$ by numerical integration of equation (B4) with an ensemble-averaged $\Delta \tau(\rho, \theta)$ obtained by averaging 10,000 realizations of the stress field aligned on their nucleation point (or, approximately, on their maxima). We then apply the arrest criterion (B1) to estimate the final radius $a$. Figure 9 shows a satisfactory comparison between this approach (open circles), the dynamic simulations based on the ensemble-averaged stress (black triangles) and the average trends of the dynamic simulations based on individual realizations of the stress field. In the remainder of this appendix we quantify analytically the conditions for the earthquake size transition.

[76] A crack that has grown considerably larger than the correlation length of the heterogeneous stress field can be modeled by a crack subjected to uniform loading by the average stress drop $\langle\Delta \tau\rangle$ plus a pair of concentrated forces $F$ representing the excess of stress drop in the nucleation area. We can obtain $F$ numerically from our ensemble- 
averaged stress field (average of 10,000 realizations, aligned at maxima) as

$$
F=\iint(\Delta \tau-\langle\Delta \tau\rangle) d S
$$

Alternatively, if we assume $a_{c} \gg a_{v}$, the shape of the mean stress field can be approximated by the autocorrelation function of stress $C$, normalized by $\operatorname{std}^{2}$, and we can write

$$
F=(\Delta \tau(0)-\langle\Delta \tau\rangle) \iint C(x, z) d S
$$

where $\Delta \tau(0)$ is the stress drop at the hypocenter. Defining the integral above as $c(H) a_{c}^{2}$, with $c(H)$ being dimensionless, and again using $a_{c} \gg a_{v}$ to assume $\Delta \tau(0) \approx \tau_{s}-\tau_{d}$, we can write

$$
F=\left(\tau_{s}-\tau_{d}-\langle\Delta \tau\rangle\right) c(H) a_{c}^{2} .
$$

For the circular mode I crack with radius $a$ the average stress intensity factor resulting from $F$ and $\langle\Delta \tau\rangle$ is

$$
K_{0}=\frac{F}{(\pi a)^{3 / 2}}+2\langle\Delta \tau\rangle \sqrt{\frac{a}{\pi}}
$$

The two terms in (B8) have competing contributions, the first decreases whereas the second increases as a function of $a$. We find that $K_{0}$ reaches a minimum

$$
K_{\min }=8\left(\frac{F\langle\Delta \tau\rangle^{3}}{2(3 \pi)^{3}}\right)^{1 / 4},
$$

at the radius

$$
a_{\min }=\sqrt{\frac{3 F}{2 \pi\langle\Delta \tau\rangle}} .
$$

For a stable equilibrium crack to exist we need

$$
K_{\min } \leq K_{c}=\sqrt{2 \mu G_{c} /(1-\nu)},
$$

which yields the following condition:

$$
F\langle\Delta \tau\rangle^{3} \leq \frac{(3 \pi)^{3}}{2^{11}} K_{c}^{4} \approx 0.41 K_{c}^{4} .
$$

Combining with (B7) the condition above can be written in terms of $\langle\Delta \tau\rangle$ as

$$
\left(\tau_{s}-\tau_{d}-\langle\Delta \tau\rangle\right)\langle\Delta \tau\rangle^{3} \leq \frac{(3 \pi)^{3}}{2^{11}} \frac{K_{c}^{4}}{c(H) a_{c}^{2}} .
$$

When (B13) is violated, the fault tends to generate runaway ruptures. Introducing the nucleation half length of an in-plane rupture,

$$
a_{\nu}=\frac{1.158}{2} \frac{\mu}{1-\nu} \frac{D_{c}}{\tau_{s}-\tau_{d}},
$$

it can then be shown that the critical $\langle\Delta \tau\rangle_{c}$ and $a_{\min }$ are given by

$$
\langle\Delta \tau\rangle_{c} \approx\left(\tau_{s}-\tau_{d}\right)\left[\frac{(3 \pi)^{3}}{2^{9} 1.158^{2} c(H)} \frac{a_{\nu}{ }^{2}}{a_{c}^{2}}\right]^{1 / 3}
$$

and

$$
a_{\min , c} \approx \frac{2}{\pi} a_{c}\left[\frac{1.158 c(H)^{2} a_{c}}{a_{\nu}}\right]^{1 / 3},
$$

respectively. Considering the amount of simplifying assumptions involved in the derivations above, these estimates are in fair agreement with the results of our dynamic simulations.

[77] If fracture energy scales with crack size as $G_{c} \approx$ $G_{c}^{0}+\gamma a$, it can be shown, by similar arguments to those above, that a sharp transition to runaway rupture exists at average stresses of order $\sqrt{(\pi \mu \gamma) /(2(1-\nu))}$, which can be higher than in the constant $G_{c}$ case.

\section{Appendix C: Estimation of Radiated Energy}

[78] As shown by Kostrov [1974] and recently summarized by Rivera and Kanamori [2005], the radiated energy $E_{r}$ can be expressed through surface integrals over the fault plane:

$$
\begin{aligned}
E_{r}= & \frac{1}{2} \int_{\Sigma}\left(\tau_{i j}^{1}-\tau_{i j}^{0}\right) \Delta u_{i} \nu_{j} d S-\int_{\Sigma} 2 \gamma_{\mathrm{eff}} d S \\
& -\int_{t_{0}}^{t_{1}} d t \int_{\Sigma(t)}\left(\tau_{i j}-\tau_{i j}^{0}\right) \Delta \dot{u}_{i} \nu_{j} d S
\end{aligned}
$$

Here, $\Sigma$ is the fault plane, and $\Sigma(t)$ is the ruptured fault surface at time $t$ up to the trailing edge of the process zone. The unit normal vector to the fault plane is $v_{j}$. Initial and final stress states are denoted by $\tau_{i j}^{0}$ and $\tau_{i j}^{1}$, respectively; and $\Delta u$ and $\Delta \dot{u}$ are slip and slip velocity. The effective fracture energy is $\gamma_{\text {eff }}$ and $t_{0}$ and $t_{1}$ are times before and after the earthquake.

[79] Since the slip velocity $\Delta \dot{u}$ is zero outside the currently rupturing zone $\Sigma(t)$, we can evaluate the last integral over the whole fault plane. By doing this, we also include the process zone and thus the second term in equation (C1). So what we are actually evaluating is

$$
\begin{aligned}
E_{r}= & \frac{1}{2} \int_{\Sigma}\left(\tau_{i j}^{1}-\tau_{i j}^{0}\right) \Delta u_{i} \nu_{j} d S \\
& -\int_{t_{0}}^{t_{1}} d t \int_{\Sigma}\left(\tau_{i j}-\tau_{i j}^{0}\right) \Delta \dot{u}_{i} \nu_{j} d S .
\end{aligned}
$$

This form is more convenient for numerical evaluation than the one proposed by Favreau and Archuleta [2003], which is obtained from $(\mathrm{C} 2)$ by integration by parts of the second term. Because we are only simulating pure strike-slip events, we further neglect the downdip slip components. We therefore obtain the following simplified expression for $E_{r}$, 
which can be conveniently evaluated numerically from the quantities available during our simulations:

$$
\begin{aligned}
E_{r}= & \frac{1}{2} \iint_{x, z}\left(\tau^{1}-\tau^{0}\right) \Delta u^{1} d x d z \\
& -\int_{t} d t \iint_{x, z}\left(\tau-\tau^{0}\right) \Delta \dot{u} d x d z .
\end{aligned}
$$

[80] Acknowledgments. We thank S. Ide and R. Archuleta for their detailed and constructive reviews. They also provided challenging comments, some of which require future improvements and research beyond the present paper. We also wish to thank the Associate Editor J. Townend for carefully inspecting the manuscript and providing additional suggestions for improvement. We thank E. Dunham for providing us his spectral boundary integral code "MDSBI." J.-P. Ampuero is supported by SPICE, a Marie Curie Research Training Network in the 6th Framework Program of the European Commission. This is contribution 1448 of the Institute of Geophysics, ETH Zurich.

\section{References}

Abercrombie, R. E. (1995), Earthquake source scaling relationships from -1 to $5 \mathrm{M}_{\mathrm{L}}$ using seismograms recorded at $2.5-\mathrm{km}$ depth, J. Geophys. Res., 100(B12), 24,015-24,036.

Abercrombie, R. E., and J. R. Rice (2005), Can observations of earthquake scaling constrain slip-weakening?, Geophys. J. Int., 162, 406-424, doi:10.1111/j.1365-246X.2005.02579.x.

Allen, R. M., and H. Kanamori (2003), The potential for earthquake early warning in southern California, Science, 300, 786-789.

Ampuero, J. P., and J. P. Vilotte (2003), Effective fault friction and seismic nucleation phase: Scale-dependent or non-linear friction?, Geophys. Res Abstr., 5, 11288

Ampuero, J.-P., J.-P. Vilotte, and F. J. Sānchez-Sesma (2002), Nucleation of rupture under slip dependent friction law: Simple models of faul zone, J. Geophys. Res., 107(B12), 2324, doi:10.1029/2001JB000452.

Ampuero, J. P., J. Ripperger, and P. M. Mai (2006), Properties of dynamic earthquake ruptures with heterogeneous stress drop, in Earthquakes: Radiated Energy and the Physics of Faulting, Geophys. Monogr Ser., vol. 170, edited by R. Abercrombie et al., pp. 255-261, AGU, Washington, D. C.

Andrews, D. J. (1980), A stochastic fault model 1. Static case, J. Geophys Res., 85, 3867-3877.

Andrews, D. J. (1981), A stochastic fault model 2. Time-dependent case, J. Geophys. Res., 86, 10,821-10,834.

Andrews, D. J. (2005), Rupture dynamics with energy loss outside the slip zone, J. Geophys. Res., 110, B01307, doi:10.1029/2004JB003191.

Aochi, H., and S. Ide (2004), Numerical study on multi-scaling earthquake rupture, Geophys. Res. Lett., 31, L02606, doi:10.1029/2003GL018708.

Beroza, G. C., and T. Mikumo (1996), Short slip duration in dynamic rupture in the presence of heterogeneous fault properties, J. Geophys. Res., 101, 22,449-22,460.

Beroza, G. C., and P. Spudich (1988), Linearized inversion for fault rupture behavior: Application to the 1984 Morgan Hill, California, earthquake, J. Geophys. Res., 93, 6275-6296.

Bouchon, M. (1997), The state of stress on some faults of the San Andreas system as inferred from near-field strong motion data, J. Geophys. Res., $102,11,731-11,744$

Brune, J. N. (1970), Tectonic stress and the spectra of seismic shear waves from earthquakes, J. Geophys. Res., 75, 4997-5009.

Campillo, M., and I. R. Ionescu (1997), Initiation of antiplane shear instability under slip dependent friction, J. Geophys. Res., 102, 20,363-20,371

Cochard, A., J. P. Ampuero, and J. Schmittbuhl (2006), Seismic nucleation under nonlinear slip weakening friction, Geophys. Res. Abstr., 8, EGU06A- 07,176

Cochran, E. S., J. E. Vidale, and S. Tanaka (2004), Earth tides can trigger shallow thrust fault earthquakes, Science, 306, 1164-1166, doi:10.1126/ science.1103,961

Dahlen, F. A. (1974), On the ratio of P-wave to S-wave corner frequencies for shallow earthquake sources, Bull. Seismol. Soc. Am., 64(4), 1159-1180.

Day, S. M. (1982), Three-dimensional simulation of spontaneous rupture: The effect of nonuniform prestress, Bull. Seismol. Soc. Am., 72(6), 1881 1902.

Delouis, B., D. Giardini, P. Lundgren, and J. Salichon (2002), Joint inversion of InSAR, GPS, teleseismic, and strong-motion data for the spatial and temporal distribution of earthquake slip: Application to the 1999 Izmit mainshock, Bull. Seismol. Soc. Am., 92(1), 278-299.
Dunham, E. M. (2005), Dissipative interface waves and the transient response of a three-dimensional sliding interface with Coulomb friction, J. Mech. Phys. Sol., 53, 327-357, doi:10.1016/j.jmps.2004.07.003.

Dyskin, A. V. (1999), On the role of stress fluctuations in brittle fracture, Int. J. Fract., 100, 29-53.

Fabrikant, V. I. (1989), Applications of Potential Theory in Mechanics, Elsevier, New York.

Favreau, P., and R. J. Archuleta (2003), Direct seismic energy modeling and application to the 1979 Imperial Valley earthquake, Geophys. Res. Lett., 30(5), 1198, doi:10.1029/2002GL015968.

Favreau, P., M. Campillo, and I. R. Ionescu (2002), Initiation of shear instability in three-dimensional elastodynamics, J. Geophys. Res., 107(B7), 2147, doi:10.1029/2001JB000448.

Frankel, A. (1991), High-frequency spectral falloff of earthquakes, fractal dimension of complex rupture, $b$ value, and the scaling of strength on faults, J. Geophys. Res., 96, 6291-6302.

Fukuyama, E., and R. Madariaga (2000), Dynamic propagation and interaction of a rupture front on a planar fault, Pure Appl. Geophys., 157, $1959-1979$

Gallovic, F., and J. Brokesova (2004), On strong ground motion synthesis with $\mathrm{k}^{-2}$ slip distributions, J. Seismol., 8, 211-224.

Geubelle, P. H., and J. R. Rice (1995), A spectral method for three-dimensional elastodynamic fracture problems, J. Mech. Phys. Solids, 43(11), $1791-1824$

Hartzell, S. H., and T. H. Heaton (1983), Inversion of strong-ground motion and teleseismic waveform data for the fault rupture history of the 1979 Imperial Valley, California, earthquake, Bull. Seismol. Soc. Am., 73 , $1553-1583$

Heaton, T. H. (1990), Evidence for and implications of self-healing pulses of slip in earthquake rupture, Phys. Earth Planet. Inter., 64, 1-20.

Herrero, A., and P. Bernard (1994), A kinematic self-similar rupture process for earthquakes, Bull. Seismol. Soc. Am., 84(4), 1216-1228.

Hillers, G., Y. Ben-Zion, and P. M. Mai (2006), Seismicity on a fault controlled by rate- and state-dependent friction with spatial variations of the critical slip distance, J. Geophys. Res., 111, B01403, doi:10.1029/ 2005JB003859.

Hillers, G., P. M. Mai, Y. Ben-Zion, and J. P. Ampuero (2007), Statistical properties of seismicity of fault zones at different evolutionary stages, Geophys. J. Int., in press.

Ide, S., and H. Aochi (2005), Earthquakes as multiscale dynamic ruptures with heterogeneous fracture surface energy, J. Geophys. Res., 110, B11303, doi:10.1029/2004JB003591

Ide, S., and M. Takeo (1997), Determination of constitutive relations of fault slip based on seismic wave analysis, J. Geophys. Res., 102, 27,37927,391 .

Johnson, E. (1990), On the initiation of unidirectional slip, Geophys. J. Int., $101,125-132$.

Kanamori, H., and D. L. Anderson (1975), Theoretical basis of some empirical relations in seismology, Bull. Seismol. Soc. Am., 65, 10731095.

Kostrov, B. V. (1974), Seismic moment and energy of earthquakes, and seismic flow of rock, Izv. Earth Phys., (1), 23-40.

Lai, Y.-S., A. B. Movchan, and G. J. Rodin (2002), A study of quasicircular cracks, Int. J. Fract., 113, 1-25.

Lavallée, D., and R. J. Archuleta (2003), Stochastic modeling of slip spatial complexities for the 1979 Imperial Valley, California, earthquake, Geophys. Res. Lett., 30(5), 1245, doi:10.1029/2002GL015839.

Lavallée, D., P. Liu, and R. J. Archuleta (2006), Stochastic model of heterogeneity in earthquake slip spatial distributions, Geophys. J. Int., 165, 622-640, doi:10.1111/j.1365-246X.2006.02943.x

Liu-Zeng, J., T. Heaton, and C. DiCaprio (2005), The effect of slip variability on earthquake slip-length scaling, Geophys. J. Int., 162, 841-849, doi:10.1111/j.1365-246X.2005.02679.x.

Madariaga, R. (1977), High-frequency radiation from crack (stress drop) models of earthquake faulting, Geophys. J. R. Astron. Soc., 51, 625-651.

Madariaga, R. (1983), High-frequency radiation from dynamic earthquake fault models, Ann. Geophys., 1, 17-23.

Mai, P. M., and G. C. Beroza (2002), A spatial random field model to characterize complexity in earthquake slip, J. Geophys. Res., 107(B11), 2308, doi:10.1029/2001JB000588.

Mai, P. M., P. Spudich, and J. Boatwright (2005), Hypocenter locations in finite-source rupture models, Bull. Seismol. Soc. Am., 95(3), 965-980, doi: $10.1785 / 0120040,111$

Mai, P. M., P. Somerville, A. Pitarka, L. Dalguer, S. Song, G. Beroza, H. Miyake, and K. Irikura (2006), On scaling of fracture energy and stress drop in dynamic rupture models: Consequences for near-source ground-motions, in Earthquakes: Radiated Energy and the Physics of Faulting, Geophys. Monogr. Ser., vol. 170, edited by R. Abercrombie et al., pp. 283-293, AGU, Washington, D. C. 
McGarr, A., J. B. Fletcher, and N. M. Beeler (2004), Attempting to bridge the gap between laboratory and seismic estimates of fracture energy, Geophys. Res. Lett., 31, L14606, doi:10.1029/2004GL020091.

McGuire, J. J., L. Zhao, and T. H. Jordan (2002), Predominance of unilateral ruptures for a global catalog of large earthquakes, Bull. Seismol. Soc. Am., 92(8), 3309-3317.

Miyatake, T. (1992a), Reconstruction of dynamic rupture process of an earthquake with constraints of kinematic parameters, Geophys. Res. Lett., $19,349-352$.

Miyatake, T. (1992b), Dynamic rupture process of inland earthquakes in Japan: Weak and strong asperities, Geophys. Res. Lett., 19, $1041-$ 1044.

Nielsen, S. B., and K. B. Olsen (2000), Constraints on stress and friction from dynamic rupture models of the 1994 Northridge, California, earthquake, Pure Appl. Geophys., 157, 2029-2046.

Oglesby, D. D., and S. M. Day (2002), Stochastic fault stress: Implications for fault dynamics and ground motion, Bull. Seismol. Soc. Am., 92(8), 3006-3021.

Ohnaka, M. (2003), A constitutive scaling law and a unified comprehension for frictional slip failure, shear fracture of intact rock, and earthquake rupture, J. Geophys. Res., 108(B2), 2080, doi:10.1029/2000JB000123.

Olsen, K. B., R. Madariaga, and R. J. Archuleta (1997), Three-dimensional dynamic simulation of the 1992 Landers earthquake, Science, 278, 834838.

Olson, E. L., and R. M. Allen (2005), The deterministic nature of earthquake rupture, Nature, 438, 212-215, doi:10.1038/nature04214.

Peyrat, S., K. Olsen, and R. Madariaga (2001), Dynamic modeling of the 1992 Landers earthquake, J. Geophys. Res., 106, 26,467-26,482.

Rivera, L., and H. Kanamori (2005), Representations of the radiated energy in earthquakes, Geophys. J. Int., 162, 148-155, doi:10.1111/j.1365246X.2005.02648.x.

Sekiguchi, H., K. Irikura, T. Iwata, Y. Kakehi, and M. Hoshiba (1996), Determination of the location of faulting beneath Kobe during the 1995 Hyogo-ken Nanbu, Japan, earthquake from near-source particle motion, Geophys. Res. Lett., 23(4), 387-390.

Shakal, A., H. Haddadi, V. Graizer, K. Lin, and M. Huang (2006), Some key features of the strong-motion data from the M 6.0 Parkfield, California, earthquake of 28 September 2004, Bull. Seismol. Soc. Am., 96(4b), S90-S118, doi:10.1785/0120050,817.
Smith, D. E. (2006), A new paradigm for interpreting stress inversions from focal mechanisms: How 3D stress heterogeneity biases the inversions toward the stress rate, Ph.D. thesis, Calif. Inst. of Technol., Pasadena.

Uenishi, K., and J. R. Rice (2003), Universal nucleation length for slipweakening rupture instability under nonuniform fault loading, J. Geophys. Res., 108(B1), 2042, doi:10.1029/2001JB001681.

Uenishi, K., and J. R. Rice (2004), Three-dimensional rupture instability of a slip-weakening fault under heterogeneous loading, Eos Trans. $A G U$, 85(47), Fall Meet. Suppl., Abstract S13E-04.

Venkataraman, A., G. C. Beroza, S. Ide, K. Imanishi, H. Ito, and Y. Iio (2006), Measurements of spectral similarity for microearthquakes in western Nagano, Japan, J. Geophys. Res., 111, B03303, doi:10.1029/ 2005JB003834.

Voss, R. F. (1988), Fractals in nature: From characterization to simulation, in The Science of Fractal Images, edited by H. O. Peitgen and D. Saupe, pp. $21-70$, Springer, New York.

Wald, D., and T. Heaton (1994), Spatial and temporal distribution of slip for the 1992 Landers, California, earthquake, Bull. Seismol. Soc. Am., 84, $668-691$.

Wells, D. L., and K. J. Coppersmith (1994), New empirical relationships among magnitude, rupture length, rupture width, rupture area, and surface displacement, Bull. Seismol. Soc. Am., 84(4), 974-1002.

Wesnousky, S. G. (1988), Seismological and structural evolution of strikeslip faults, Nature, 335, 340-342.

Zeng, Y., J. G. Anderson, and G. Yu (1994), A composite source model for computing realistic synthetic strong ground motions, Geophys. Res. Lett., 21(8), 725-728.

Zhang, W., T. Iwata, K. Irikura, H. Sekiguchi, and M. Bouchon (2003), Heterogeneous distribution of the dynamic source parameters of the 1999 Chi-Chi, Taiwan, earthquake, J. Geophys. Res., 108(B5), 2232, doi:10.1029/2002JB001889.

J.-P. Ampuero, D. Giardini, P. M. Mai, and J. Ripperger, Institute of Geophysics, ETH Zurich, Schafmattstr. 30, CH-8093 Zurich, Switzerland (ripperger@sed.ethz.ch) 